\title{
Evidence for methane segregation at the surface of Pluto
}

\author{
S. Douté ${ }^{\prime}$ and B. Schmitt \\ Laboruloire de Gluciologie et Géophysique de l'Environnement, 54 rue Molière, BP 96, 38402 Si-Martin-d'Hères, Frunce \\ $T e l: 33476824239$ \\ $F_{4 x}: 33476824201$ \\ E-mail : sylvain@gluciog.ujf-grenoble.fr \\ E. Quirico ${ }^{2}$ \\ Luborusire de Gkaciologie et Géophysique de l'Environnement \\ T. C. Owen ${ }^{3}$ \\ Instiute for Astronomy, University of Huwaii, 2680 Woodluwn Dr. Honolulu, HJ 96822, USA \\ D. P. Cruikshank ${ }^{3}$ \\ NASA Ames Reseurch Center, MS 245-6 Moffet: Field, CA 94035-1000, USA \\ C. de Bergh ${ }^{3}$ \\ Observutoire de Puris, 5 Pluce Jules Junssen 92195 Meudon, France \\ T. R. Geballe ${ }^{4}$ \\ Joint Astronómy Center, 660 North A'ohoku Pluce, Universiry Purk, Hilo, Henwuï, 96720, USA \\ T. L. Roush \\ Sun Froncisco Sture Univ. And NASA Ames Reseurch Center, MS 245-6 Moffet Field, CA 94035-1000, USA \\ 'New address : Institute of Geophysics and Plenetury Physics, UCLA, 405 Hilgard, Box 951567, Los Angeles, CA 90095 \\ ${ }^{2}$ New address : Institut d'Astrophysique Spuriale, Bat 121, Université Puris XI, 91405 Orsuy, Frunce \\ ${ }^{3}$ Guest observer, United Kingdom Infrured Telescope, Joint Astronomy Center, Hawaii, USA. \\ ¿New uddress : Gemini Observulory, 670 Nonh A'ohoku Pluce, Universiry Purk, Hilo, HI 96720, USA.
}

Number of manuscript pages (Including Tables and captions) : 46

Number of figures : 14

Number of tables : 4

KEY WORDS : Pluto, Surface ; Ices ; Spectrophotometry ;Charon ; Radiative transfer 
Running Head : $\mathrm{CH}_{4}$ SEGREGATION ON PLUTO

Correspondence : Sylvain Douté,

Institute of Geophysics and Planetary Physics,

UCLA, 405 Hilgard, Box 951567

Los Angeles, CA 90095, USA

E-mail: sdoute@igpp.ucla.edu 
Abstract

In May 1995, a set of spectrophotometric curves of the system Pluto-Charon was recorded with the UKIRT telescope equipped with the spectrometer CGS4. As for the previous observations by Owen ef al. (1993), the spectra cover a part of the near infrared range, between 1.4 and $2.55 \mu \mathrm{m}$, bul with a higher resolution of approximately 700. In both the 1992 (Owen et al. 1993) and 1995 data, the existence of solid methane is confirmed by numerous absorption bands, and the carbon monoxide and the nitrogen ices are identified by their respective signatures at 2.35 and $2.15 \mu \mathrm{m}$. The solid nitrogen seems to be the principal icy component and forms a matrix in which the $\mathrm{CH}_{4}$ and $\mathrm{CO}$ molecules are diluted. However a spectroscopic analysis of the 1995 observations indicates that pure methane may coexist with its diluted phase in $\mathrm{N}_{2}$. In order to derive the horizontal and vertical distribution of these different species and to obtain some quantitative information about their characteristics, we have modeled the spectrum of May 15 that corresponds to the maximum of Pluto's visible light curve. This was achieved by means of a radiative transfer algorithm dealing with compact and stratified media (Douté and Schmitt 1998). Among the various representations we have tested to describe the surface of Pluto, only a geographical mixture of three distinct units explains all the significant structures of the analyzed spectrum. The first unit is a thin granular layer of pure $\mathrm{CH}_{4}$ covering a compact polycrystalline substratum of $\mathrm{N}_{2}-\mathrm{CH}_{4}-\mathrm{CO}$, which are in a molecular mixture (concentrations of $\mathrm{CH}_{4}$ and $\mathrm{CO}$ of the order of $0.45 \%, 0.1-0.2 \%$ respectively). It covers about $70 \%$ of the observed area and corresponds to volatile deposits that are sublimating under solar illumination. The second unit is either (a) a single thick layer of pure granular methane or (b) a unit similar to the first unit but with the two components inverted (i.e. with $\mathrm{CH}$, forming a substratum and the $\mathrm{N}_{2}-\mathrm{CH}_{4}-\mathrm{CO}$ mixture a superficial layer of fine grains). Covering $20 \%$ of the surface, it represents some old surfaces that have been sublimated for a long time, and eventually recovered later by very small amounts of fresh deposits of the molecular mixture $\mathrm{N}_{2}-\mathrm{CH}_{4}-\mathrm{CO}$. Finally, the third unit may result from the condensation of very fine grains of nearly pure $\mathrm{N}_{2}$. It covers the remainder of the surface (about $10 \%$ ). All these results allow a better understanding of the processes of deposition, metamorphism, sublimation and transport affecting the different ices detected on Pluto during its climatic cycles. 


\section{INTRODUCTION}

Pluto, the ninth and the last identified planet of the solar system remains the only one that has not yet been visited by a spacecraft. However, our knowledge about this object has significantly progressed during the last few years thanks to recent instrumental developments.

For the first time, the treatment of HST (Hubble Space Telescope) images, produced in late June and early July 1994 by the FOC (Faint Object Camera, ESA) in blue light has allowed Stern et al. (1997) to generate maps nearly covering the two hemispheres of Pluto with $160 \mathrm{~km}$ resolution. These maps show a huge spatial variability of the visible albedo with a dozen distinctive features, including a bright polar cap. The features are thought to be mainly related to changes of the chemical and physical properties over the surface.

In the same way, the improvement of ground-based near-infrared (NR) and to a less extent visible (Grundy and Fink 1996) spectrophotometry, has dramatically increased our capabilities to determine surface properties. This new generation of observations has come essentially from UKIRT (United Kingdom InfraRed Telescope, Mauna Kea, Hawaii). The initial measurements were made in 1992 with the NIR spectrometer CGS4 (Cooled Grating Spectrometer) and covered the 1.4 to $2.5 \mu \mathrm{m}$ range at a spectral resolution of $6.7 \mathrm{~nm}$ (Owen et al. 1993). They included the light reflected by both the spatially unresolved disks of Pluto and Charon. A spectroscopic analysis of the different reflectance spectra obtained has provided crucial information about the nature and the physical state of the materials that cover the uppermost millimeters or centimeters of the surface of Pluto. The presence of solid methane was confirmed by eight characteristic absorption bands. In addition, carbon monoxide and nitrogen ices were identified by their respective signatures at 2.35 
and $2.15 \mu \mathrm{m}$. Carbon dioxide, an ice that is detected on Triton, seems to be absent on Pluto, as well as hydrocarbons like $\mathrm{C}_{2} \mathrm{H}_{2}, \mathrm{C}_{2} \mathrm{H}_{4}, \mathrm{C}_{2} \mathrm{H}_{6}, \ldots$ but water ice cannot be excluded (Owen et al. 1993).

This study also showed that the methane molecules are most probably diluted in nitrogen ice but not as diluted as on Triton. This was deduced by noting that the position and width of most of the methane bands fit those of a laboratory sample of $1 \% \mathrm{CH}_{4}$ mixed in $\mathrm{N}_{2}$ better than those of pure $\mathrm{CH}_{4}$ ice on one side, or those of $\mathrm{CH}_{4}$ even more highly diluted in $\mathrm{N}_{2}$, on the other side (Schmitt et al. 1992, Quirico 1995). The higher mean $\mathrm{CH}_{4}$ concentration (2.6\%) deduced by the model for Pluto compared to Triton also pointed toward an intermediate state of mixing. However, the laboratory data available at that time were insufficient to define this state more precisely. Subsequent extensive laboratory studies on pure $\mathrm{CH}_{4}$ and $\mathrm{CO}$ (as well as several other molecules) and their various mixtures with solid nitrogen (Quirico and Schmitt 1997a,b), Quirico et al. 1996, 1998, Schmitt et al. 1998) allowed a detailed spectroscopic analysis of the initial 1992 UKIRT observation as well as a set of spectra taken in 1993. This led to the suggestion that a segregation of methane with an enriched top layer possibly occurs at the surface of Pluto (Schmitt et al. 1994, Quirico 1995) rather than a horizontal distribution of patches of pure and diluted $\mathrm{CH}_{4}$ (Cruikshank et al. 1997, Grundy and Fink 1996).

The analysis of the profile of the $2.15 \mu \mathrm{m}$ band of nitrogen, used as a «thermometer » in the 1993 observations, led Tryka et al. (1994) to propose a value of $40 \pm 2 \mathrm{~K}$ for the temperature of nitrogen ice on Pluto.

For Charon, water ice is the only compound that was recognized unambiguously by its spectral signatures at 1.55 and $2.04 \mu \mathrm{m}$ (Buie et al. 1987), but other components may also exist (e.g. Roush 1994). 
In May 1995, an additional series of three Pluto-Charon observations was acquired with the UKIRT-CGS4 system in the same spectral range but with a spectral resolution improved by a factor of about $2.5(2.64 \mathrm{~nm})$. In this paper, we present a spectroscopic analysis of these observations as well as numerical modeling of one of these spectra. The scope of this work is a first attempt to derive the horizontal and vertical distribution modes of the different ices detected, in order to solve the problem raised above. This study also leads to more quantitative information about the intrinsic chemical and physical properties (composition, grain sizes, ...) and about the geographical extend of the solids identified so far. In the first part of the paper, the observational data is described as well as the relevant calibration procedures. The second part is dedicated to the analysis of the Pluto spectrum. First, we recall the contributions of the modeling approach, and present the framework and the numerical tool used for our simulations. Second, after modeling and extracting the contribution of Charon to the measured spectral reflectance, we assign the different bands observed and analyze the methane bands in terms of the physical state in which the methane occurs. We then i describe how we tested and selected the best surface representations corresponding to the different coexistence modes of the molecular mixture $\mathrm{N}_{2}-\mathrm{CH}_{4}-\mathrm{CO}$ and pure methane suggested by the spectroscopic analysis. The synthesis of the previous results, followed by their interpretation and discussion is presented in the final part of the paper.

\section{PRESENTATION AND CALIBRATION OF THE OBSERVATIONS}

In May 1995, three non-spatially resolved reflectance spectra of the Pluto-Charon system were acquired respectively on May 11, 13 and 15. The longitudes of the sub-earth point on the surface of Pluto were respectively $66^{\circ}, 313^{\circ}$ and $200^{\circ}$. 
The CGS4 spectrometer measures the flux received by the UKIRT telescope with a spectral resolution of $2.64 \mathrm{~nm}$ (resolving power $\lambda / \mathrm{d} \lambda$ of approximately 750 ). The 1.4-2.1 $\mu \mathrm{m} \mathrm{H}$ range and the 1.9-2.55 $\mu \mathrm{m} \mathrm{K}$ range were recorded separately. The measurement of the lines of an argon lamp and the observation of the atmospheric bands of the $\mathrm{OH}$ radical, both in emission, ensure a good spectral wavelength calibration with a mean uncertainty of $\pm 1 \mathrm{~nm}$. The details of the calibration procedure are described in Quirico et al. (1998).

The radiative power which arrives on the detectors is integrated for one hour on the average. The resulting curve $S(\lambda)$, measured in digital units, is deeply marked by absorptions due to the transfer through the terrestrial atmosphere. As a consequence, a spectrum $S_{\bullet}(\lambda)$ of a solar type star is recorded in the same region of the sky with only a short time shift. Because $S \cdot(\lambda)$ is affected by the same air mass, it allows us to correct these disturbing atmospheric bands (principally those of $\mathrm{CO}_{2}$ and $\mathrm{H}_{2} \mathrm{O}$ ). Unfortunately, it does not insure a satisfactory radiometric calibration. For that, another reference measurement $S_{c a}(\lambda)$ is performed on a star whose near-infrared flux $F_{c a l}(\lambda)$ is well known but also disturbed by telluric absorptions. By the elimination from $S_{c a l}(\lambda)$ of the disturbing signatures which can be easily extracted in that case, one obtains its envelope $S_{\text {cal }}^{E}(\lambda)$ which is used for the calibration. The first quantity to be calibrated that way is the flux of the solar type star which can be written :

$$
F_{*}(\lambda)=\frac{F_{c o l}(\lambda)}{S_{c o l}^{E}(\lambda)} S_{*}(\lambda)
$$

Then dividing the radiance $\mathrm{B}_{\mathrm{T}}(\lambda)$ of a black body with a similar temperature by this flux determines an atmospheric correcting factor: 


$$
\kappa(\lambda)=\frac{\mathrm{B}_{T}(\lambda)}{\frac{\mathrm{F}_{\text {col }}(\lambda)}{\mathrm{S}_{\text {rol }}^{E}(\lambda)} \mathrm{S}_{*}(\lambda)}
$$

The equivalence between the solar type star and the corresponding reference black body can be written :

$$
\frac{S_{*}^{E}(\lambda)}{S_{c a l}^{E}(\lambda)}=\frac{B_{T}(\lambda)}{F_{c a l}(\lambda)} \Rightarrow B_{T}(\lambda)=\frac{S_{*}^{E}(\lambda)}{S_{c a l}^{E}(\lambda)} F_{c a l}(\lambda)
$$

$S_{*}^{E}(\lambda)$ being the envelope of the spectrum of the solar type star. The calibration operation continues with the multiplication of the spectral observed signal $S(\lambda)$ coming from Pluto-Charon by the correcting factor $\kappa(\lambda)$, along with its transformation into a flux :

$$
F(\lambda)=\frac{S(\lambda)}{S_{*}(\lambda)} \frac{S_{*}^{E}(\lambda)}{S_{c a l}^{E}(\lambda)} F_{c a l}(\lambda)
$$

Finally, the astronomical spectrum is converted into a geometrical albedo $A_{g}$ according to the expression :

$$
A_{8}(\lambda)=\frac{D^{2} d^{2}}{R^{2}} \frac{F(\lambda)}{F_{S}(\lambda)}
$$

where $D$ and $d$ respectively correspond to the heliocentric and the geocentric distance of PlutoCharon, $R$ their mean quadratic radius, $F(\lambda)$ the corrected and calibrated flux as received on Earth and $F_{S}(\lambda)$ the solar flux at $1 \mathrm{AU}$, as tabulated by Smith and Gottlieb (1974).

After applying this calibration procedure, it appeared that no photometric coherence is achieved between the $\mathrm{H}$ and $\mathrm{K}$ spectral regions as judged from the overlapping 1.9-2.1 $\mu \mathrm{m}$ range. In the $\mathrm{K}$ region all three measurements seem to give consistent albedo spectra while the $\mathrm{H}$ region gives unexpectedly high and variable values. On the May 11, clouds attenuated the signal from the calibration star (BS5384) while its H band spectrum was being measured. On May the 13 and 15 , the skies were clear, but we observed the star at $\mathrm{H}$ through a partially opened dome because of 
concerns that the starlight would saturate the array of the CGS4 spectrometer. Following the data reduction procedures described above, we multiplied the $\mathrm{H}$ band spectra from the three nights by scaling factors $(0.75,0.47$, and 0.375 for the 11,13 and 15 May, respectively) in order to match the $\mathrm{H}$ and $\mathrm{K}$ band spectra in their regions of overlap. We note that the scaled $\mathrm{K}$ band fluxes are consistent with those measured in earlier years when a wider slit was used and a better photometrical calibration achieved. For example, a K band spectrum measured on April 16, 1994, corresponds to the visible light curve minimum of Pluto at $96^{\circ}$ longitude close to that of May 11 , $1995\left(66^{\circ}\right)$. On this curve, their corresponding visible albedos differ by a bit less than $5 \%$ (Fig. 1), (Buie et al. 1997). It appears that the fluxes measured over the $\mathrm{K}$ region are very consistent and that the scaled flux of the $\mathrm{H}$ spectrum of May 11, 1995, also fit most of the corresponding range of April 16, 1994 quite well (Fig. 2). In addition, a H spectrum recorded on May 27, 1992 at $51^{\circ}$ longitude, i.e. only $15^{\circ}$ (and $4 \%$ visible albedo) away from the $05 / 11 / 95$ observation, also fits the scaled $95 \mathrm{H}$ spectrum within a few percents. Nevertheless, part of the 2 surrounding spectra does not match exactly with the 95 measurement, essentially outside the strong methane bands. The noticeable difference in observation conditions $\left(-15^{\circ}\right.$ and $+30^{\circ}$ for the 92 and 94 spectra relative to the 95 one) as well as a possible real small evolution of the surface of Pluto in terms of average surface composition and/or physical state, may explain the spectral differences.

In conclusion, we believe that our initial photometric calibration is correct for all three spectra in the $\mathrm{K}$ region and after rescaling for the $\mathrm{H}$ region spectra. Knowing that the 1992 and 1994 reference spectra at lower resolution display an estimated uncertainty of $\pm 15 \%$, we think that our calibration operation yields all three H\&K May 1995 flux spectra within $\pm 20 \%$. In the following we will use the calibrated and concatenated 1.4-2.55 $\mu \mathrm{m}$ flux spectra, except for May 13 restricted to the $\mathrm{H}$ region due to some spurious effects in the $\mathrm{K}$ region data. 


\section{ANALYSIS OF THE OBSERVATION}

\subsection{Framework of the modeling approach}

In the visible and near infrared ranges, the flux that comes to the earth from the Pluto-Charon system or from any other planetary body in general, totally or partly arises from the reflection and the absorption of the solar light which penetrates more or less deeply into the surface. The intimate interaction with the constituent materials implies that their physico-chemical and structural properties potentially mark the spectral signal. Moreover, as light attenuation into the materials often changes by several orders of magnitude at different wavelengths, every reflectance level of a spectrum probes a particular range of depths. If it is modulated enough, the general shape of the spectrum then becomes very sensitive to the occurrence of a stratification of the surface properties. For all these reasons, beyond a simple identification of the chemical nature and of the physical state of the components, as expressed by the precise absorption band positions (see part 3.3), the modeling of the intensity and the shape of the bands is the only efficient way to obtain additional information (see part 3.4). These are mainly the chemical composition of the compounds and the texture, as well as the granularity of the materials they form. These properties can be obtained as a function of depth in the case of surface stratification.

However things can be a bit more complicated. Most of the time different kinds of uniform surface units, which form something like a more or less regular geographical mosaic that is not spatially resolved by the observation, may contribute to the same spectral signal. Then, they linearly add their respective contributions according to their surface proportion and the viewing geometry. This kind of addition is called a 'geographical mixture'. This is always the case for a spectrophotometric 
measurement, like the one we analyzed, because it integrates the light reflected by the whole hemisphere of the planetary body of interest.

For the modeling of the spectrum of the Pluto-Charon system, we used an efficient radiative transfer algorithm calculating the spectral bidirectional reflectance of an icy or mineral stratified surface (Douté and Schmitt 1998). Each layer of such a surface can present a granular or a compact texture. The aim of the algorithm is to determine, for any kind of illumination and viewing conditions, the link between the physical, chemical and structural properties of the underlying medium and its observed spectral signature. A potential stratification is described by the vertical superposition of any number of plane-parallel homogeneous layers.

Practically, the algorithm is integrated into a software that can globally simulate and then analyze by interactive inversion hyperspectral data according to a wide variety of observational and surface situations (Douté 1998). Indeed this system can deal with spectrophotometric data (as is the case here with Pluto-Charon), and with hyperspectral images, both corresponding to surfaces that can present any kind of chemical composition, geographical and vertical distribution of the materials. The modeling is performed by the integration of the radiative spectral power reflected by the different surface units that generally constitute a planetary surface. The previous radiative transfer algorithm ensures the calculation of each contribution of such a geographical mixture. All the above mentioned characteristics lead to a very powerful system to reach our main objective : to test and select different surface representations of Pluto.

\subsection{Modeling and extraction of the contribution of Charon}

We first need to separate the respective contributions of Pluto and of its satellite. The problem consists of extracting a spectrum of Charon from our observation which was acquired at a far 
higher resolution $(\mathrm{d} \lambda=2.64 \mathrm{~nm})$ than any available Charon spectrum. Roush et al. (1996) derived the spectral geometric albedo of Charon from very poorly resolved $(\mathrm{d} \lambda=0.1 \mu \mathrm{m})$ spectrophotometric data acquired between 1.5 and $2.5 \mu \mathrm{m}$ during a special mutual event : the total eclipse of Charon by Pluto on April 23, 1987 (Buie et al. 1987). We first modeled this geometric albedo curve with high resolution simulations deresolved at $d \lambda=0.1 \mu \mathrm{m}$ (Fig. 3). For that, we have to choose a suitable, but simplified, surface representation of Charon. The simplest one we found is a uniform hemisphere covered by a geographical mixture of two kinds of surface units :

- a unit $U_{w}$, which is a granular optically semi-infinite layer of water ice, - a unit $U_{n}$, which is spectrally neutral and of unknown chemical nature.

For the first unit, intended to reproduce the absorption bands, we use new optical constants of crystalline water ice at $40 \mathrm{~K}$ (Grundy and Schmitt 1998). The second unit permits the $2 \mu \mathrm{m}$ flux of the strong $\mathrm{H}_{2} \mathrm{O}$ bands to rise to the correct level. This was impossible to realize with a hemisphere entirely covered with $\mathrm{H}_{2} \mathrm{O}$ grains. The principal parameters inferred by the inversion (surface proportion of the water ice $\Phi_{\mathrm{H} 2 \mathrm{O}}^{\text {surf }}=60 \%$, mean grain size $d_{\mathrm{H} 2 \mathrm{O}}=50 \mu \mathrm{m}$ ) as well as the chosen surface representation may not be very significant, nor well constrained, but the deresolved spectrum they generate by direct modeling is within the error bars of the geometric albedo of Charon.

Using the previous values, we calculate the corresponding high resolution ( $d \lambda=2.64 \mathrm{~nm}$ ) spectrum of Charon which is directly transformed into flux using Eq. 4. This flux contribution is then removed from the Pluto-Charon 1995 observations. We finally convert the resulting spectrum of Pluto back to geometric albedo thanks again to Eq. 5 (Fig. 4).

As the eclipse observation of Charon corresponds to a Pluto central longitude of $190^{\circ}$, the removal of its spectral contribution is strictly valid only for the May 15, 1995 observation (200 ${ }^{\circ}$ Pluto 
longitude). Near-infrared spectral variations with Charon longitude have been observed and attributed to changes in water ice absorption (Bosh et al. 1992, Buie and Shriver 1994), so the removal of the eclipse observation from the May 11 or 13, 1995 observations may lead to incorrect subtraction of the water ice signature from Charon. For that reason, in this paper we restricted our modeling effort to the May 15 observation. On the other hand, the following spectroscopic analysis of the two other Pluto spectra (at $66^{\circ}$ and $313^{\circ}$ ), mainly based on $\mathrm{CH}_{4}$ band positions, should not be significantly altered by the first order removal of Charon water ice absorption using its $190^{\circ}$ synthetic spectrum.

\subsection{Spectroscopic analysis}

After some discussion of band assignments we will present the spectral analysis applied to the higher resolution spectra described in this paper.

\subsubsection{Band assignment}

The high resolution spectra of Pluto exhibit 20 bands in the 1.4-2.5 $\mu \mathrm{m}$ range. About half of them were observed and assigned by Owen et al. (1993) to $\mathrm{N}_{2}, \mathrm{CH}_{4}$ and $\mathrm{CO}$. We complete this identification and indicate, for each band, the specific vibration mode of the corresponding molecule (Fig. 5a,b). In particular, the observation of the second overtone of solid CO at 1.579 $\mu \mathrm{m}$ confirms the identification of this molecule on Pluto. The band at $1.69 \mu \mathrm{m}$ that we attribute to pure solid $\mathrm{CH}_{4}$ is a special case discussed in more detail below.

Compared to Triton, there is no positive sign of the 3 strong $\mathrm{CO}_{2}$ ice bands around $2 \mu \mathrm{m}$, thus excluding the presence of this molecule in significant amount at the surface of Pluto. The problem of water ice is more complicated because its broad absorption bands combined with the broad $\mathrm{CH}_{4}$ bands make its direct identification very difficult. As an illustration, just looking at 
the spectrum of the Pluto-Charon system displayed in Figure 4, it is not evident that it contains the signature of water ice, although this component actually contributes to at least $13 \%$ of the projected surface! This point will be further addressed by the detailed modeling of the spectrum (see 3.4), but only for the observation simulated in this paper (5/15/95).

Only one band that is present in all 3 of the May 1995 observations, but not observable in the former lower resolution observations, cannot be attributed to one of the three molecules identified so far on Pluto. It peaks around $1.75 \mu \mathrm{m}$, and is slightly shifted from one observation to the other in the $1.749-1.752 \mu \mathrm{m}$ range. This band has also been observed in the spectrum of Triton where it has been suggested that ethane $\left(\mathrm{C}_{2} \mathrm{H}_{6}\right)$, or perhaps propane $\left(\mathrm{C}_{3} \mathrm{H}_{8}\right)$ diluted in solid nitrogen may be potential candidates (Quirico et al. 1998). Comparisons with the available laboratory data on various molecules (Quirico and Schmitt 1997a, Quirico et al. 1998) show that pure $\mathrm{C}_{2} \mathrm{H}_{2}, \mathrm{C}_{2} \mathrm{H}_{4}, \mathrm{C}_{2} \mathrm{H}_{6}, \mathrm{C}_{3} \mathrm{H}_{8}, \mathrm{HCN}, \mathrm{HC}_{3} \mathrm{~N}, \mathrm{CH}_{3} \mathrm{OH}, \mathrm{SO}_{2}, \mathrm{NO}, \mathrm{NO}_{2}$ and $\mathrm{NH}_{3}$ ices cannot explain this band but that $\mathrm{C}_{2} \mathrm{H}_{6}$ and possibly $\mathrm{C}_{3} \mathrm{H}_{8}$ diluted in solid nitrogen display a strong band only shifted by $0.003-0.006 \mu \mathrm{m}$ from the observed feature. However, the limited relevance of the currently available data on both molecules (measured at $1 \%$ concentration in $\alpha-\mathrm{N}_{2}$ at $21 \mathrm{~K}$ ) does not explain the remaining shift, thus still precluding a firm identification. Laboratory data on these molecules at more relevant temperatures and concentrations are clearly needed.

\subsubsection{Physical state of the $\mathrm{CH}_{4}$ molecules}

Using the laboratory data of Quirico and Schmitt (1997a), we can compare the methane bands in the Pluto spectra with those of pure solid methane around $40 \mathrm{~K}$ and those of $\mathrm{CH}_{4}$ diluted in solid $\beta-\mathrm{N}_{2}$ around this same temperature (Fig.. $6 \mathrm{a}, \mathrm{b}$ ). The first striking point is that the weak, but real, band present in all 3 observations around $1.69 \mu \mathrm{m}$ is only active in pure solid methane. 
Indeed, this band has never been observed in any sample of $\mathrm{CH}_{4}$ diluted in $\mathrm{N}_{2}$ (neither in $\alpha$ or $\beta$ $\mathrm{N}_{2}$ phases) and at any temperature. This band is clearly a witness of the presence of pure methane at the surface of Pluto.

The second point is that the peak of the strongest bands in the $\mathrm{K}$ region (at 2.20, 2.32, 2.37, and $2.43 \mu \mathrm{m})$, particularly for the 15 May spectrum, are situated at positions intermediate between pure and diluted methane, contrary to the same absorptions in the spectrum of Triton that perfectly fit the positions of diluted $\mathrm{CH}_{4}$ (Fig. 4b). These bands are also much wider, partly due to saturation of the bands (at 2.32 and $2.37 \mu \mathrm{m}$ ) but possibly also due to the simultaneous contribution of both forms of methane. If we now look at the medium bands in the $\mathrm{H}$ region, they more closely fit the wavelength of diluted methane for the 11 May observation, but they are still slightly shifted towards low frequency compared to Triton in the 15 May, and particularly in the 13 May spectra (Fig. 6a). In addition the side band near $1.78 \mu \mathrm{m}$ is less pronounced than on Triton, indicating a possible contribution of pure methane, partly filling the trough between the side and the main band. Finally the two weak bands around 1.48 and $1.95 \mu \mathrm{m}$ follow similar trends, the 11 and 15 May bands being at intermediate positions and the 13 May bands almost fitting the positions of pure methane.

All this information points toward the coexistence of pure and diluted methane at the surface of Pluto. For the 15 May observation the apparent increase of the diluted character of methane when looking at bands with decreasing strengths, probing deeper into the surface, also suggests that at least part of the pure methane should be preferentially accumulated near the surface. The reverse seems to occur for the 11 May spectrum, pure methane showing up at depth (weak bands) and diluted methane dominating the top layers. The effective occurence of such vertical stratifications, 
and of any other type of $\mathrm{CH}_{4}$ segregation, should be confirmed by a detailed modeling of the whole spectrum for various possible surface representations (see 3.4).

It should be noted that the phase diagram of the binary $\mathrm{N}_{2}-\mathrm{CH}_{4}$ mixture (Prokhvatilov and Yantsevich 1983) tells us that absolutely pure methane ice cannot coexist with nitrogen ice, but that a small fraction of nitrogen $(3-4 \%$ around $40 \mathrm{~K})$ should be diluted in it. The positions of the $\mathrm{CH}_{4}$ bands in such a system are slightly shifted from the positions for pure $\mathrm{CH}_{4}$ ice, as shown by laboratory spectra (Quirico et al. 1996, Schmitt et al. 1998). However this shift is smaller than the resolution of the observations and does not affect our interpretation. In the following we will continue to refer to this $\mathrm{CH}_{4}$-rich mixture containing a few percent nitrogen as "pure methane".

These intermediate positions of the $\mathrm{CH}_{4}$ bands provide important information about the physical state of $\mathrm{CH}_{4}$ at the surface of Pluto. On the other hand, they preclude the extraction of any information or constraint on the temperature of the $\mathrm{N}_{2}-\mathrm{CH}_{4}-\mathrm{CO}$ mixture and on the crystalline phase of solid $\mathrm{N}_{2}$ based on the precise position of the $\mathrm{CH}_{4}$ bands, as was possible for Triton where only diluted $\mathrm{CH}_{4}$ is present (Quirico et al. 1998). In particular, the most sensitive band relative to the phase of nitrogen is strongly perturbed by the suspected contribution of pure $\mathrm{CH}_{4}$ ice. The detailed modeling of the shape of the $\mathrm{CH}_{4}$ bands may.help constrain the surface temperature, but it is anticipated that the blending between the two $\mathrm{CH}_{4}$ contributions will dominate the spectrum. The $\mathrm{CO}$ bands being weak and quite insensitive to temperature and phase, the only remaining temperature tracer seems to be the weak nitrogen band at $2.15 \mu \mathrm{m}$. As has been noted before, its broad shape is clearly indicative of the predominance of the $\beta$ phase, i.e. a temperature larger than $35.6 \mathrm{~K}$. But only a thorough modeling of the shape of the weak $\mathrm{N}_{2}$ band together with the underlying $\mathrm{CH}_{4}$ band may provide some additional constraint on the temperature of the nitrogenrich deposits. 


\subsection{Test and selection of the best surface representations}

\subsubsection{Presentation}

The previous spectroscopic analysis suggests the coexistence of two phases of methane on the surface of Pluto : the pure solid form and methane diluted in solid nitrogen. Three simple potential modes of coexistence can be imagined: (I.) a granular (intimate) mixture, (II.) a vertical stratification, and (III.) a geographical mixture. An intimate mixture of grains respectively formed by pure methane and « $\mathrm{CH}_{4}$ contaminated » nitrogen is the first possibility (1.). A second possibility (II.) would be a vertical segregation of pure $\mathrm{CH}_{4}$ and of a $\mathrm{N}_{2}-\mathrm{CH}_{4}-\mathrm{CO}$ molecular mixture. The third possibility (III.) is that more or less extended horizontal patches of pure and diluted $\mathrm{CH}_{4}$ coexist on the surface of Pluto. Nevertheless, as shown in 3.3.2, these basic surface representations will have to be combined in different ways in order to provide the best solutions for the problem of the species distribution. Due to the geographically integrated nature of the available spectra, it is not yet possible to derive a precise spatial distribution of the different physico-chemical units we can expect at the surface of Pluto. Consequently, we propose a crude idealization of the three basic representations of differentiation we have described, each covering the entire visible hemisphere of Pluto :

\section{First basic representation $R i$ (intimate)}

A uniform deposit composed of one single thick layer :

- a unit $U_{i}$ formed by a homogeneous population of pure $\mathrm{CH}_{4}$ and $\mathrm{N}_{2}-\mathrm{CH}_{4}-\mathrm{CO}$ grains intimately mixed $\left(G_{c}\right.$ and $G_{m}$, respectively).

II. Second basic representation Rs (stratified)

A stratified and uniform deposit $\mathrm{U}_{\mathrm{c} / \mathrm{m}}$ composed of two superimposed distinct layers : 
- a superficial layer $L_{c}$ of finite depth, formed by a homogeneous population of pure $\mathrm{CH}_{4}$ grains.

- an optically semi-infinite substratum $\mathrm{L}_{m}$, formed by a homogeneous population of $\mathrm{N}_{2}-\mathrm{CH}_{4}-$ $\mathrm{CO}$ (molecular mixture) grains.

\section{Third basic representation $\mathrm{Rg}$ (geographic)}

A uniform geographical 'mosaic' of two distinct units :

- a unit $\mathrm{U}_{c}$ formed by an homogeneous population of pure $\mathrm{CH}_{4}$ grains.

- a unit $\mathrm{U}_{\mathrm{m}}$ formed by an homogeneous population of $\mathrm{N}_{2}-\mathrm{CH}_{4}-\mathrm{CO}$ (molecular mixture) grains.

These three configurations imply three different lists of free parameters for the models, which are listed in TABLE I. In the previous and all the following notations of the «Units », $U$, the subscript «c » refers to pure $\mathrm{CH}_{4}$, «m » to the $\mathrm{N}_{2}-\mathrm{CH}_{4}-\mathrm{CO}$ molecular mixture, « $\mathrm{m}+\mathrm{c}$ » (aliased to « $\mathrm{i}$ » for the « Representations », R) to the intimate (granular) mixtures of « $c$ » and « $m$ » grains, «c/m »

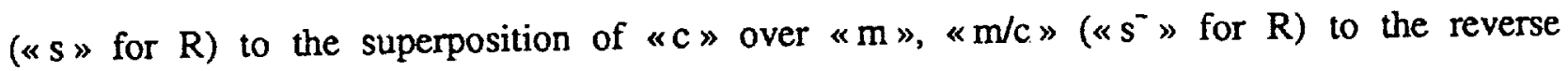
stratification, and « $\mathrm{n} »$ refers to the neutral or fine grained $\mathrm{N}_{2}$ unit. All surface representations (except the basic representation $R_{g} \equiv R_{c m}$ ) that is a geographical combination of two or more of these units will be described by the concatenation of the corresponding subscripts $\left(e . g .: R_{i 5 n} \equiv U_{c+m}\right.$ $\left.+\mathrm{U}_{\mathrm{c} / \mathrm{m}}+\mathrm{U}_{\mathrm{n}}\right)$

The first difficulty that arises when modeling the spectrum of Pluto with either basic surface representations $R_{i}, R_{s}$, or $R_{g}$, is an obvious impossibility to fit the albedo level for the 2.32 and the $2.37 \mu \mathrm{m} \mathrm{CH}_{4}$ bands. Indeed, due to the required saturation of these two bands, the models always lead to albedos much lower than observed. There are two possibilities to increase the albedo: 
- add a new geographical unit that will contribute most of the flux at 2.32 and $2.37 \mu \mathrm{m}$. This implies a minimum level of brightness for the constituent materials.

- mix at least part of the surface grains with some other particles that have to be bright enough (high single scattering albedo) to increase the reflectance around 2.32 and $2.37 \mu \mathrm{m}$ in particular.

In both cases, the additional necessary component(s) should have an almost neutral spectral reflectance over the entire range, since we do not observe any significant continuum slope in the spectrum and only a single weak unidentified localized signature (possibly linked with units containing $\mathrm{CH}_{4}$, if the ethane identification is confirmed). Among the molecules that have been identified in the spectrum of Pluto, only nitrogen, even a with small amount of $\mathrm{CH}_{4}$ and $\mathrm{CO}$, can present this neutrality, if it is present as grains that are sufficiently fine. This property comes from the weakness and the uniqueness of the $\mathrm{N}_{2}$ absorption band at $2.15 \mu \mathrm{m}$. We choose to add a new unit of fine grained nitrogen ice to the geographical mosaic with the other compounds rather than forming a granular mixture of $\mathrm{N}_{2}$-rich grains within the unit $\mathrm{U}_{\mathrm{m}}$, as it is thermodynamically very improbable to have an intimate mixture of two very different grain sizes of the same volatile material. In addition, because of the non-linear behavior of a granular mixture, it is necessary to introduce an extremely large quantity of fine grained nitrogen to produce a substantial rise of the reflectivity at 2.32 or $2.37 \mu \mathrm{m}$ where methane is highly absorbing, whatever its state of dilution (pure or diluted).

Consequently, we add to all our future representations based on $R_{i}, R_{s}$ and $R_{g}$ a spectrally neutral geographical unit $U_{n}$ that is described by the following parameters :

$\mathrm{U}_{\mathrm{n}}$ [fine grains of nitrogen]

- surface proportion $\mathcal{P}^{\mathrm{surf}}{ }_{n}$

- mean grain size $r_{n}$ 
- scattering parameter $g_{n}$.

One can note that several scattering parameters ' $\mathrm{g}$ ' appear in the previous list and in TABLE I. They express the angular distribution of the scattered power by a volume element of a layer and are related to the Henyey-Greenstein phase function as defined and illustrated by Hapke (1993). They cannot really be constrained by a directionally integrated measurement as is the case for our spectrum of Pluto. Without any additional information, we decided to set all these parameters to zero (isotropic scattering grains). This particular choice may have a noticeable effect on the values of the parameters closely related to $\mathrm{g}$, i.e. mainly the grain sizes, but not on their orders of magnitude. For the other parameters, the results of the inversion should be relatively insensitive to our choice.

In order to calculate the scattering and the absorption properties of the materials that constitute the different layers of the surface units, the radiative transfer model we use for the analysis needs their optical indices versus :wavelength. The spectroscopic analysis has given some information about the physical state of the identified components : $\mathrm{N}_{2}, \mathrm{CH}_{4}$, and $\mathrm{CO}$ (see part 3.3). Consequently, we select a set of optical indices, measured in the laboratory and compatible with these constraints. Below, we describe these data, ice by ice :

- Pure $\mathrm{CH}_{4}$ ice : we use a set of measurements performed at $40 \mathrm{~K}$ (unpublished) and similar to the data at $24 \mathrm{~K}$ and $30 \mathrm{~K}$ (Quirico and Schmitt 1997a, Schmitt et al. 1998). The effects of temperature principally affect the position and the width of the bands (as well as the continuum), but remain weak in these data, especially when considering the resolution of the Pluto observations. Thus, the coherence of the optical index file is insured if the effective surface temperature of $\mathrm{CH}_{4}$ ice is not too different from $40 \mathrm{~K}$. This may be the case because 
temperatures slightly higher (up to $54 \mathrm{~K}$ ) than the average are expected for this kind of deposit due to its lower volatility than $\mathrm{N}_{2}$ ice (Stansbeny et al. 1996).

- Pure $\mathrm{N}_{2}$ ice : the laboratory data we use were obtained in the $\beta$ phase at $36.5 \mathrm{~K}$, slightly above the $\alpha-\beta$ phase transition, by Grundy et al. (1993) and Quirico 1995.

- Molecular mixture $\mathrm{N}_{2}-\mathrm{CH}_{4}-\mathrm{CO}$ : it is not possible to experimentally obtain the optical index spectra corresponding to all the potential values for the concentrations $\mathrm{C}_{\mathrm{CH}}$ and $\mathrm{C}_{\mathrm{OO}}$ in solid nitrogen. As a consequence, we numerically generated the data needed from a sequence of experimental measurements concerning $\mathrm{N}_{2}-\mathrm{CH}_{4}$ and $\mathrm{N}_{2}$ - $\mathrm{CO}$ mixtures at $36.5 \mathrm{~K}$ obtained by Quirico and Schmitt (1997a,b). For that purpose, we considered three real or virtual 'pure' reference components. The first one is identified with pure $\mathrm{N}_{2}$ ice, the second and the third ones with methane and carbon monoxide initially diluted in the nitrogen matrix, but artificially normalized to unit concentration (see Quirico et al. 1998). Then we assume that, for each wavelength, the scalar product of the complex indices of $\mathrm{N}_{2}$, of the normalized diluted $\mathrm{CH}_{4}$ and of the normalized diluted $\mathrm{CO}$ by the vector $\left[\left(1-\mathrm{C}_{\mathrm{CH} 4}-\mathrm{C}_{\mathrm{CO}}\right), \mathrm{C}_{\mathrm{CH} 4}, \mathrm{C}_{\mathrm{CO}}\right]$ gives the optical constants of the mixture. The normalization and scalar product operations do not change the spectral effects linked to the dilution. Indeed the slight high frequency shifts of the absorption bands of $\mathrm{CH}_{4}$ diluted in $\beta \mathrm{N}_{2}$ compared to pure $\mathrm{CH}_{4}$ (4 to $6 \mathrm{~nm}$ in the $\mathrm{H}$ and $\mathrm{K}$ regions) strongly determine the modeling of the astronomical spectrum of Pluto.

The inversion mode we use is an interactive trial-and-error sequence of direct modeling that progressively improves the fit of the spectrophotometric curve of Pluto. For that purpose we try to reproduce the main and more significant spectral characteristics of the spectrum. These spectral characteristics are the shape, the intensity and the position of the principal absorption bands of methane, nitrogen and carbon monoxide, as well as the presence or the absence of very distinctive 
weak structures, all related to $\mathrm{CH}_{4}$ in its two phases. Aside from the bands of $\mathrm{N}_{2}$ at $2.15 \mu \mathrm{m}$ and $\mathrm{CO}$ at $2.35 \mu \mathrm{m}$, the methane features can be arranged for physical reasons into four independent groups (see Fig. 5a,b.) :

- group one : the 4 strong bands of the $\mathrm{K}$ region at $2.2,2.32,2.37$ and $2.42 \mu \mathrm{m}$. This group also contains the slight bulge around $2.28 \mu \mathrm{m}$ standing on the high frequency wing of $2.32 \mu \mathrm{m}$ band. This feature appears to be a significant structure because it can be reproduced by the modeling.

- group two : the 3 medium bands of the $H$ region at $1.67,1.72$ and $1.79 \mu \mathrm{m}$ with, on the left wing of the first and last ones, a small side band with a position that is typical of methane diluted in nitrogen.

- group three : the weak band at $1.85 \mu \mathrm{m}$ (H region).

- group four : the little absorption of pure methane which appears at $1.69 \mu \mathrm{m}$ on the hill separating the bands at 1.67 and $1.72 \mu \mathrm{m}$.

\subsubsection{Analysis}

The first task to achieve in order to precise the horizontal and vertical distribution modes of the different detected ices is to confirm one of the main results of the spectroscopic approach : there is a methane segregation at the surface of Pluto, whatever its nature. For that, we try to simulate the Pluto spectrum with even simpler representations than the three basic ones $\left(R_{i}, R_{s}\right.$ and $\left.R_{g}\right)$, i.e., considering only one form of $\mathrm{CH}_{4}$, either pure or diluted.

The first $R_{m n}$, is a geographical mosaic of two units : one unit $U_{m}$ formed by a molecular mixture of $\mathrm{N}_{2}-\mathrm{CH}_{4}-\mathrm{CO}$ and one unit $\mathrm{U}_{n}\left(\mathrm{~N}_{2}\right)$. As is illustrated in Fig..7, the simulations do not reproduce the positions of the methane bands that are intermediate between the pure and the diluted forms. Moreover it appears impossible to simultaneously fit, even roughly, the intensity and the width of 
the $\mathrm{N}_{2}$ and all the $\mathrm{CH}_{4}$ bands. Finally the characteristic signature of pure $\mathrm{CH}_{4}$ at $1.69 \mu \mathrm{m}$ is absent. In a second simple representation, $R_{c n}$, the unit $U_{m}$ is replaced by an unit $U_{c}$ composed of pure $\mathrm{CH}_{4}$ grains. Even more critical misfits are obtained (shifts, shapes, much too strong $1.69 \mu \mathrm{m}$ band, ...) (Fig. 7). These models prove more directly that both pure and diluted methane coexist at the surface of Pluto, but they did not tell which types of differentiation occurs.

In order to discriminate among the different basic types of differentiation, we performed the second task dedicated to test a series of more or less complex surface representations taking into account these differentiations. They are divided into three families. The first and the third families are respectively composed of the granular mixture $R_{i}$ and the geographical distribution $R_{g}$, completed with the neutral unit $U_{n}$ as stipulated before. The representations of the second family contain $U_{n}$ and at least one stratified unit (generally $U_{c / m}$ ). A given representation tries to reduce the limitations of the previous one. In this paper, we choose to focus on the best fits of the Pluto spectrum we have obtained so far, with each representation. In each case, we will give a synthetic scheme of the representation, the list of the associated modeling parameters and their best fit values.

\section{a.) 'Geographical representation $R_{\text {in }}$ with a granular mixture'}

According to the phase diagram of the binary $\mathrm{N}_{2}-\mathrm{CH}_{4}$ molecular mixture (Prokhvatilov and Yantsevich 1983), the coexistence of almost pure $\mathrm{CH}_{4}$ grains (with small amount of nitrogen) and nitrogen-rich grains saturated by $\mathrm{CH}_{4}$ can occur under some temperature and mean composition conditions. If the conditions are met, the concentration of $\mathrm{CH}_{4}$ in the $\mathrm{N}_{2}$ matrix is constrained to a value of approximately $2 \%$ around $40 \mathrm{~K}$. On the other hand the $\mathrm{CO}$ concentration is not constrained by the $\mathrm{N}_{2}-\mathrm{CO}$ phase diagram. 
Taking these constraints into account, our simplest representation $R_{\text {in }}$ includes an unit $U_{n}$ and an intimate (granular) mixture of pure $\mathrm{CH}_{4}$ ice grains and $\mathrm{N}_{2}-\mathrm{CH}_{4}-\mathrm{CO}$ grains with the $\mathrm{CH}_{4}$ concentration fixed to $2 \%$ (unit $\mathrm{U}_{\mathrm{c}+\mathrm{m}}$ ). We took the optical constants of pure $\mathrm{CH}_{4}$ for the former grains, as only very small spectral changes are expected and measured for methane ice with $1-2 \%$ nitrogen diluted in it (Quirico et al. 1996; Schmitt et al. 1998). For the $2 \% \mathrm{CH}_{4}-\mathrm{N}_{2}(-\mathrm{CO})$ grains we used the values of diluted $\mathrm{CH}_{4}$ (stated limit of validity, see 3.4.1.).

The best fit of the observation by this representation is shown in Figure 8. Although a satisfactory fit of most of the $\mathrm{CH}_{4}$ bands is achieved, there are at least three misfits that cannot be solved with this representation. First the strength of the $\mathrm{N}_{2}$ band is more than an order of magnitude too weak. Increasing its strength by increasing its grain size strongly irremediably saturates the methane bands. The high (2\%) $\mathrm{CH}_{4}$ concentration in nitrogen is surely the principal reason . Secondly, the low frequency wings of the strongest $\mathrm{CH}_{4}$ bands, especially at 2.32 and $2.37 \mu \mathrm{m}$, are not well reproduced, indicating some lack of pure methane close to the surface. On the other hand, this representation has too much pure $\mathrm{CH}_{4}$ at some depth, as witnessed by the too strong $1.69 \mu \mathrm{m}$ band. All these results, obtained with a vertically homogeneous distribution of the different types of grains, point to the existence of a stratification favoring the presence of pure methane close to the surface. This leads to the next type of surface representations.

\section{b.) 'Geographical representations with stratification'}

Among this category, the selected representations of the surface of Pluto were inspired by two preliminary trials. The first one $R_{5 n}$ consists of a uniform geographical mosaic of two distinct units :

- a unit $U_{c / m}$ associated with the basic vertical distribution $R_{s}$, (a layer of pure $\mathrm{CH}_{4}$ grains over a $\mathrm{N}_{2}-\mathrm{CH}_{4}-\mathrm{CO}$ mixture), 
- a neutral unit $\mathrm{U}_{\mathrm{n}}$ composed with fine grains of nitrogen.

The second trial, $R_{s}^{-} n$, is similar to the first one but the two layers of the unit $U_{d m}$ are inverted giving $U_{m / c}$. Both simulations reproduce the main features of the spectrum quite well but do not show all the finest details at the same time (the bulge around $2.28 \mu \mathrm{m}$, the side bands in the $\mathrm{H}$ region and the absorption of pure methane at $1.69 \mu \mathrm{m})$. Nevertheless they tell us that, if a stratification of the deposits really occurs, pure methane should lie at the surface but also at some depth. This conclusion leads to the first retained representation :

\section{i) Representation $R_{s c n}$}

A uniform geographical mosaic of three distinct units :

- a unit like $\mathrm{U}_{\mathrm{c} / \mathrm{m}}$ of pure $\mathrm{CH}_{4}$ grains stratified over a $\mathrm{N}_{2}-\mathrm{CH}_{4}-\mathrm{CO}$ molecular mixture,

- a unit $\mathrm{U}_{c}$ formed by a homogeneous population of pure $\mathrm{CH}_{4}$ grains,

- a unit $U_{n}$ of fine spectrally neutral grains of nitrogen.

TABLE II contains a synthetic scheme of this representation, the list of the associated parameters as well as the values derived from the best modeling of the spectrum. Figure 9 . illustrates the quality of the fit obtained between the observed and the synthetic spectra (see also Fig. 12a,b).

In the $\mathrm{K}$ region (Fig. 12b), the spectral position, the width and the shape of the methane bands at 2.20, $2.32 \mu \mathrm{m}$ and to a lesser extent at 2.37 and $2.43 \mu \mathrm{m}$ are quite well reproduced. In particular, we can notice that the slight bulge around $2.28 \mu \mathrm{m}$ is fit well by the synthetic spectrum. The absorption of nitrogen at $2.15 \mu \mathrm{m}$ and of carbon monoxide at $2.35 \mu \mathrm{m}$ correctly mark their presence by two small spikes. However, the asymmetry that affects the wings of $\mathrm{CO}$ band is reversed. If now, we move to the $\mathrm{H}$ region (Fig. 12a), the simulated spectrum reproduces the global shape of the intermediate bands of $\mathrm{CH}_{4}$, with the exception of the intensities of the 1.67 and $1.72 \mu \mathrm{m}$ bands which are slightly too strong. The small side bands around 1.65 and $1.78 \mu \mathrm{m}$ clearly stand out and 
coincide rather well with the observation. On the other hand, we can notice that the small absorption spike of pure methane at $1.69 \mu \mathrm{m}$ is present but a bit too weak. The shape and particularly the width and intensity of the $1.85 \mu \mathrm{m}$ band are not well fitted, but between 1.82 and $1.95 \mu \mathrm{m}$ imperfect cancellation of telluric absorptions probably occurred in the spectrum of Pluto. Finally the continuum around $1.5,2$ and $2.5 \mu \mathrm{m}$ is not well reproduced, although the 4 weak $\mathrm{CH}_{4}$ bands at about $1.45,1.48,1.93$ and $1.97 \mu \mathrm{m}$ are satisfactorily simulated. However we know that in these ranges of very weak absorption, our optical constants of $\mathrm{CH}_{4}$ diluted in nitrogen are very uncertain and noisy (see Quirico et al. 1998).

\section{ii) Representation $R_{s s} n$}

For the second selected representation, we consider a uniform geographical mosaic of three distinct units :

- a unit $\mathrm{U}_{c / m}\left(\mathrm{CH}_{4}\right.$ stratified over a $\mathrm{N}_{2}-\mathrm{CH}_{4}-\mathrm{CO}$ molecular mixture),

- a second unit $U_{m / c}$ similar to the previous one but with the layers $L_{c}$ and $L_{m}$ inverted $\left(N_{2}-C_{4}\right.$ $\mathrm{CO}$ over pure $\mathrm{CH}_{4}$ ),

- a third unit $U_{n}$ of fine spectrally neutral grains of nitrogen.

TABLE III displays a synthetic scheme of the $R_{s s}-$ representation, the list of the model parameters as well as their best fit values. Figure 10. shows the best fit obtained between the observed and simulated spectra (see also Fig. 12a,b).

There are very few differences between this simulation and $R_{s c n}$, whose qualities seem to be globally equivalent. We only notice, in the $\mathrm{H}$ region, the improvement of the intensity for the intermediate methane bands and the unfortunately correlated degradation of the intensities of the two side bands. The fit of the hill around $1.75 \mu \mathrm{m}$ also deteriorates a little bit. 
It should be mentioned that, among the different parameters which are used in the current representation, the concentrations $C^{2} \mathrm{CH}_{4}$ and $C^{2} \mathrm{Co}$ as well as the mean grain size $\mathrm{r}_{\mathrm{m} 2}$ of the molecular mixture superficial layer of the unit $U_{m / c}$ are not well constrained. Indeed, this layer has a limited influence on the weak $\mathrm{N}_{2}$ band, which is essentially produced and controlled by the substratum of the unit $U_{c / m}$. We can be much more confident of the parameter values obtained for the lastmentioned unit $\mathrm{U}_{\mathrm{c} / \mathrm{m}}$.

We will not proceed further with the stratified surface representations and keep the two best fitting simulations we have obtained so far.

\section{c.) 'Geographical representation $R_{\text {mcn }}$ without stratification'}

In order to test if a stratification really has to be invoked, we first study a simple geographical distribution of pure methane and methane diluted in $\mathrm{N}_{2}$ (with $\mathrm{CO}$ ).

We thus consider a uniform geographical mosaic of three distinct units :

- a unit $\mathrm{U}_{\mathrm{m}}$ formed by a homogeneous population of $\mathrm{N}_{2}-\mathrm{CH}_{4}-\mathrm{CO}$ (molecular mixture) grains,

- a unit $\mathrm{U}_{c}$ of volatile deposits made of a homogeneous population of pure $\mathrm{CH}_{4}$ grains,

- a third unit $U_{n}$ of fine spectrally neutral grains of pure nitrogen.

In fact, $R_{m c n}$ correspond to the basic representation $R_{g}$ plus the neutral unit $U_{n}$. The synthetic scheme of the representation, the list of the associated parameters as well as the best fit values are contained in TABLE IV. Fig 11. illustrates the quality of the fit reached between the observed and synthetic spectra (see also Fig. 13a,b).

The global quality of this simulation comes close to the level of precision displayed by the previous modeling $R_{s c n}$ and $R_{s s}{ }_{n}^{-}$but without reaching it completely. If we exclusively concentrate on the noticeable differences between $R_{s s} n$ and $R_{m c n}$, we can say that the latter fits the troughs on 
each side of the $2.42 \mu \mathrm{m}$ band of methane better but not the strong saturated absorptions at 2.32 and $2.37 \mu \mathrm{m}$, which are too broad (Fig. 13b). In addition, the band at $2.32 \mu \mathrm{m}$ does not correctly display the characteristic bulge around $2.28 \mu \mathrm{m}$, which is a significant feature. The signature at $2.2 \mu \mathrm{m}$ is also a bit too strong and wide. If we now pay attention to the nitrogen and the carbon monoxide

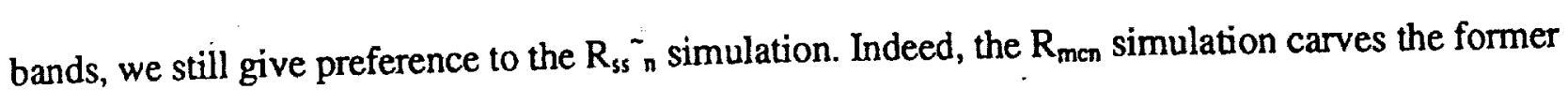
and lowers the latter a bit too much. On the other hand, the spectrum $R_{m e n}$ more longer fits the observation toward the continuum below $2.14 \mu \mathrm{m}$ than the representation $R_{s s ~} n$. In the $H$ region (Fig. 13a), the agreement of the $\mathrm{R}_{\text {men }}$ simulation appears to be significantly less satisfactory if one refers to all the significant $\mathrm{CH}_{4}$ spectral features described by the groups 2,3 and 4 in paragraph 3.4.1 (too narrow $\mathrm{CH}_{4}$ bands with less good relative intensities).

We also tested a slightly more complicated representation, $\mathrm{R}_{\mathrm{imn}}$, by replacing the pure $\mathrm{CH}_{4}$ unit $U_{c}$ of $R_{m c n}$ by an intimate mixture $U_{c+m}$. This did not significantly improve the fit, especially at the (too) strong bump around $2.28 \mu \mathrm{m}$.

\subsubsection{Synthesis}

The series of simulations we have performed clearly allow us to select as the best representations $R_{s c n}$ and $R_{s s} n$, which are characterized by the presence of one or two stratified units. However, situations without any stratification, composed of a geographical mixture of three distinct units (pure $\mathrm{CH}_{4}$, molecular mixture $\mathrm{N}_{2}-\mathrm{CH}_{4}-\mathrm{CO}$ and fine grains of nitrogen), cannot be excluded even if they lead to less satisfactory fits of the observed spectrum. For both kinds of simulations we point out that :

- a pure methane / diluted methane segregation appears to be necessary, whether it is vertical and horizontal or purely horizontal, but not granular. 
- the areas with at least the superficial layer composed of pure methane always represent an important fraction of the whole surface of the visible hemisphere of Pluto $(88,69$ and $46 \%$ for $R_{s c n}, R_{s s}$ and $R_{m c n}$ respectively). They are characterized by a granular texture of relatively small grains (a few hundred micrometers).

- the concentrations of methane and carbon monoxide diluted in nitrogen are about four times higher on Pluto than on Triton, despite the similarities between the two bodies (Quirico et al. 1998).

- the huge mean grain size (of the order of several centimeters) that is obtained for the molecular mixture $\mathrm{N}_{2}-\mathrm{CH}_{4}-\mathrm{CO}$ when it forms an optically semi-infinite substratum probably is not real. But it indicates that these deposits present very few optical interfaces' per unit volume and therefore probably have a compact polycrystalline texture with large evolved crystals rather than an accumulation of independent particles. This kind of situation was considered by Eluszkiewicz et al. (1998).

- A spectrally neutral bright area (or one with a very small spectral slope and/or very weak features) is needed over about $12 \%$ of the surface. Pure nitrogen with fine grains (order of $10 \mu \mathrm{m}$ ) was considered here as the most straightforward candidate, but this nitrogen can in fact contain as much $\mathrm{CH}_{4}$ and $\mathrm{CO}$ as the molecular mixture unit does. Simulations with such a mixture for the $U_{n}$ unit, show that only very limited parameter changes for the 3 main surface representations are needed to recover very similar fits. Any other bright neutral candidate in the near-infrared range can be considered for this unit.

- water ice, the most widespread compound throughout the outer Solar System, does not seem to be necessary to obtain very satisfactory fits of the spectrum of Pluto near the maximum of its visible light curve (around $200^{\circ}$ Jongitude). 


\section{INTERPRETATION AND DISCUSSION}

The existence of seasonal transports of volatile molecules at the surface of Pluto has been suggested 15 years ago and first modeled by Cruikshank and Silvaggio (1980), Trafton et al. (1983) and (Stern et al. 1983). However at that time, only methane was identified and then considered in the models. The discovery of solid $\mathrm{N}_{2}$ and $\mathrm{CO}$ (Owen et al. 1993) led to consideration of nitrogen (with a trace amount of $\mathrm{CO}$ ) as the main volatile molecule driving the interhemispheric transport and methane as an «impurity » having a special behavior due to its lower volatility (Trafton 1990 , Benchkoura 1996, Stansberry et al. 1996). As a result, a possible segregation of nitrogen and methane was already predicted by Lunine and Stevenson (1985) but for the surface of Triton. Using a globally averaged seasonal model, Trafton (1990) predicted that a layer of pure $\mathrm{CH}_{4}$ only a few molecules thick should overlay the $\mathrm{N}_{2}-\mathrm{CH}_{4}$ solid solution («detailed balancing layer »). On the other hand Benchkoura (1996), using a time-dependent latitudinal model of volatile transport including differential sublimation of the $\mathrm{N}_{2}-\mathrm{CH}_{4}-\mathrm{CO}$ mixture, obtained a geographical segregation of pure methane patches located at the boundary between the substrate (water ice?) and the sublimating $\mathrm{N}_{2}$-rich polar cap. These moderately hot methane areas cover between a few percent to almost $40 \%$ of Pluto's surface during the seasonal cycle.

Finally, starting from the spectroscopic analysis of the early UKIRT/CGS4 spectra suggesting a possible vertical segregation of $\mathrm{CH}_{4}$ at the surface of Pluto (Schmitt et al. 1994, Quirico 1995), one of us performed laboratory experiments demonstrating that slow sublimation of $\mathrm{N}_{2}$ ice with low $\mathrm{CH}_{4}$ concentration $(0.2 \%)$ leads to the progressive formation of a fine grained $\mathrm{CH}_{4}$ layer on top of the sublimating $\mathrm{N}_{2}$-rich molecular mixture (see Stansberry et al. 1996). Extrapolating this experiment to Pluto's surface, Stansberry et al. (1996) considered that the continuation of the segregation process may result in an ever increasing thickness of the layer of $\mathrm{CH}_{4}$ grains, finally 
leading to thick lag deposits with higher temperatures than the volatile $\mathrm{N}_{2}$-rich molecular mixture. Modeling the vertical exchanges of methane between the surface and the atmosphere, they concluded that only a few percent of the surface covered by nearly pure and warm $(-53 \mathrm{~K})$ methane patches is sufficient to produce the high methane atmospheric abundance (1\%) required to explain its elevated temperature of about $100 \mathrm{~K}$ (Yelle and Lunine 1989, Lellouch 1994, Strobel et al. 1996).

Considering the different results presented in this paper together with some considerations from the volatile transport models described above, we propose a 'scenario', describing the different transport and segregation phenomena that affect the identified ices and which lead to the surface state as revealed by the analysis of the UKIRT-CGS4 near infrared observation (see Fig. 14). Inversely, the following scenario coupled with some numerical results from our spectrum modeling will allow us to better constrain, or discard, some processes included in the different transport models.

As witnessed by the occurrence of methane segregation on Pluto, differential sublimation and / or chemical diffusion seem to affect on one side the $\mathrm{CH}_{4}$ molecule and on the other side $\mathrm{N}_{2}$ and $\mathrm{CO}$ within the volatile deposits $\mathrm{N}_{2}-\mathrm{CH}_{4}-\mathrm{CO}$ when subjected to solar radiation. At the surface, these processes create a residue of nearly pure methane forming a crust of variable thickness and divided into small grains by the escape of the $\mathrm{N}_{2}$ and the $\mathrm{CO}$ gases or by the diffusion process. After they have been mixed and transported through the atmosphere, the gases settle as a molecular mixture by direct condensation on the coldest areas. These areas, although probably extended, cover quite a small fraction of the observed hemisphere, because the telescope essentially receives the light reflected around the subsolar point, i.e. from the well illuminated and thus sublimating surfaces. Since they are in thermodynamic equilibrium with an atmosphere containing the same molecules, 
the deposits evolve more or less rapidly by metamorphism to a compact polycrystalline texture. Finally a new segregation cycle starts when they undergo the effects of the sun again as the seasonal cycle takes place, temporally modulating the sunlight received at a given latitude. This general scenario can be made more specific if we look at the details of the results obtained for the different units of the three main representations we retained.

First, the additional complexity of the 'stratified representations' $R_{s c n}$ and $R_{s s} n$ compared to the 'geographical' one $R_{m e n}$, appears to be more consistent, both at the spectroscopic and the physical level, with the observations and the transport models. Indeed, over most of the surface, the pure methane crust overlying the $\mathrm{N}_{2}-\mathrm{CH}_{4}-\mathrm{CO}$ mixture may be thin enough to let the radiation reach the compact sublimating deposits. Using the results of the model of Benchkoura (1996), we calculate that the sublimation of a mixture with $0.4 \% \mathrm{CH}_{4}$ leads to a methane crust about $100-200 \mu \mathrm{m}$ thick over most the sublimating area. Such a layer is clearly optically thin over most of the spectrum. Second, the contribution of the areas displaying optically thick $\mathrm{CH}_{4}$ residues, wherever they are situated at the surface or at depth, also appears to be necessary. They attest to the existence of old surfaces that have sublimated for a long time (possibly during previous seasonal cycles), and eventually became covered over by small amounts of fresh deposits of the molecular mixture $\mathrm{N}_{2}$ $\mathrm{CH}_{4}-\mathrm{CO}\left(\mathrm{R}_{s s}{ }_{n}\right.$ case). We note that all our models need much bigger grains for these thick crusts than for the methane fine crust. This may result from metamorphism processes occurring in these warmer $\mathrm{CH}_{4}$ patches after the sublimation and diffusion of the underlying $\mathrm{N}_{2}$ and $\mathrm{CO}$ has stopped. This is another proof of their age. Such a situation is particularly clear in the case of the $R_{s s} n$ representation where the grain sizes differ by one order of magnitude. When it exists $\left(R_{s s}{ }^{-}\right.$case), the superficial $\mathrm{N}_{2}-\mathrm{CH}_{4}-\mathrm{CO}$ frost layer consists of grains with sizes 20 times smaller compared to the grains of the active sublimating $\mathrm{N}_{2}-\mathrm{CH}_{4}-\mathrm{CO}$ areas. This may effectively give evidence of the 
freshness of this thin deposit relative to the sublimating deposits, probably condensed several tens of years ago and strongly evolved by metamorphism and densification. The very fine grained $\mathrm{N}_{2}$ rich area should also have been deposited very recently. These grains may be linked with the micron sized grains that have been inferred for the thin clouds observed in the atmosphere of Triton (Pollack et al. 1990).

The existence of all these differentiation and stratification processes on Pluto surely arises from the larger concentrations of methane and carbon monoxide that we obtain for Pluto compared to Triton (Quirico et al. 1998). Indeed, such phenomena clearly display a limited extent or do not exist at all on Triton. On Pluto, they imply the presence of an important amount of superficial pure methane which is certainly linked, as modeled by Stansberry et al. (1996), with the detection of high $\mathrm{CH}_{4}$ concentrations in the atmosphere $(-1 \%)$ (Young 1994). Although the thin $\mathrm{CH}_{4}$ crust probably did not contribute to the upward $\mathrm{CH}_{4}$ mass flux necessary to maintain the atmospheric concentration in this model, there are large enough thick $\mathrm{CH}_{4}$ deposits (surface proportion $\sim 20 \%$ ) in both stratified representations to comply with these calculations. Indeed only 1 to $3 \%$ of surface cover by hot $\mathrm{CH}_{4}$ patches is enough. The non-detection of water ice which is only confirmed at the moment for the longitudes around the maximum of the light curve as well as the absence of $\mathrm{CO}_{2}$ ice, is another particularity of Pluto compared to Triton (Cruikshank et al. 1997, Quirico et al. 1998). As water ice is expected to be present as a rocky lithosphere by the bulk composition models (see e.g. Stern 1998), the absence of such bare surface units may mean that the amount of volatile materials, or of their derived products, is quite large on the surface. The thick $\mathrm{CH}_{4}$ crust may also hide the $\mathrm{H}_{2} \mathrm{O}$ and $\mathrm{CO}_{2}$ ices on Pluto, while the complete sublimation of the volatiles on Triton uncovers them. 
Now we can determine what the best surface representations we have derived from the analysis of the UKIRT-CGS4 near infrared data would imply for the appearance of Pluto in the visible if it was imaged by the HST as performed by Stern et al. (1997). The methane, whether it is pure or diluted, does not present any noticeable signature below $0.62 \mu \mathrm{m}$, but maybe an extremely weak residual absorption due to overtone and combination bands. Nitrogen and carbon monoxide, which are quite featureless in the near infrared, are totally transparent in the visible. Consequently, a 410 $n m$ HST image of the Pluto's disk as described by the $R_{s c n}, R_{s s} n$ or $R_{m c n}$ representations would show a quite bright surface on the average with some slightly darker regions corresponding to the compact polycrystalline $\mathrm{N}_{2}-\mathrm{CH}_{4}-\mathrm{CO}$ sublimating deposits. Indeed for the latter area, the photons travel over a much longer path length before they can exit from the surface and have then more chance to be absorbed by the residual absorption of $\mathrm{CH}_{4}$. The problem is that the dynamic range of albedo across the planet locally exceeds a factor of five ( $25 \%$ when disk-averaged) on the real HST images (Stern et al. 1997). Such a contrast cannot be explained by the exclusive occurrence of highly translucent materials like the evolved deposits of $\mathrm{N}_{2}-\mathrm{CH}_{4}-\mathrm{CO}$. The $\mathrm{CCD}$ spectra of Pluto recorded at various rotational phases also display a systematic decrease of albedo toward the blue (red slope) (Buie and Fink 1987, Fink and Di Santi 1988, Grundy and Fink 1996) that cannot be explained by the ice absorption so far. Nevertheless photochemical processes can affect these basic molecules. They may be particularly intense within the superficial crusts of pure methane, first forming ethane, possibly identified through the $1.75 \mu \mathrm{m}$ band, next larger organic molecules and then leading to a very complex mixture with some compounds strongly absorbing in the visible. The results of the HST, the CCD and UKIRT-CGS4 observations provide useful constraints on the optical properties of these unknown compounds. Their absorption coefficient should present quite high values in the visible progressively decreasing around $1.0 \mu \mathrm{m}$ from where it should have weak 
values in the NIR, contrasting by a factor of 100 at least with the value at $410 \mathrm{~nm}$. Different kinds of materials may be suitable : some hydrocarbons and nitriles displaying a high carbon to hydrogen ratio (Gougeon 1998), like organic tholins (Khare et al. 1984) or kerogen for example. But minerals can also be considered. For most organic molecules, the existence in the near-infrared of many bands of various intensity, which are unobserved, implies that their proportions on the surface of Pluto must be less than a certain threshold, maybe of the order of $0.001 \%$, given the absorption level of methane in the near infrared continuum and the $S / N$ of the spectrum. More complex mixtures, like tholin, as proposed by Grundy and Fink (1996), have optical constants consistent with our constraints. They may either replace nitrogen in the neutral unit of our representations or they may be mixed with methane in the old and thick $\mathrm{CH}_{4}$ crusts. This problem should be investigated more thoroughly by conjointly modeling the visible and the near-infrared spectra. So, we can note that the search for the dark components on Pluto, even if it is a bit constrained, remains an open question.

\section{Conclusion}

The use of a polyvalent and efficient modeling tool, applied to the analysis of high quality spectrophotometric data, allowed us to demonstrate for the first time that on the surface of Pluto there exist geographical and vertical segregations of solid methane from the other detected ices, $\mathrm{N}_{2}$ and $\mathrm{CO}$. This implies the presence of extended surfaces with superficial pure methane where an intense photo-chemistry may take place, leading to the dark material evidenced by the visible HST images. Additionally, such methane rich surfaces give a possible explanation for the large abundance of $\mathrm{CH}_{4}$ in the atmosphere. All the ices, $\mathrm{N}_{2}, \mathrm{CH}_{4}$, and $\mathrm{CO}$ seem to condense, evolve and sublimate in a complex manner during the seasonal cycle. The kind of horizontal and vertical 
distributions we have obtained, at least for one hemisphere, allowed us to give a specific description of these processes.

This work only represents the first part of an important series of analyses to be performed in the near future on observations that correspond to different rotational phases of Pluto. Such analyses will certainly reveal other interesting information, in particular concerning the geographical localization of the different surface units distinguished by the modeling of the infrared spectra. As a consequence, it will be easier to study the link between these units and the albedo features of the HST visible images whose chemical and physical nature could be then less ambiguously determined. 


\section{ACKNOWLEDGMENTS}

We wish to thank the staff of UKIRT for its support of these observations. The Joint Astronomy Centre, on behalf of the United Kingdom Particle Physics and Astronomy Research Council, operates UKIRT. 


\section{REFERENCES}

Benchkoura, A. 1996. Modélisation du transport de volatils à la surface des planètes et satellites glacés du Système Solaire. Application à Tríon et Pluton. Thesis, LGGE, University Joseph Fourier, Grenoble, France.

Bosh, A.S., Young, L.A., Elliot, J.L., Hammel, H.B. and R.L. Baron 1992. Photometric variability of Charon at 2.2 $\mu$. Icurus, 95(2), $319-324$.

Buie, M.W. and U. Fink 1987. Methane absorption variations in the spectrum of Pluto. learus, 70(3), 483-498.

Buie, M. W. Cruikshank, D. P., Lebofsky, L A., and E. F. Tedesco 1987. Water frost on Charon. Narure, 329, 522-523.

Buie, M.W. and S.K. Shriver 1994. The distribution of water frost on Charon. Icurus, 108(2). 225-233.

Buie, M. W., Tholen, D. J. and LH. Wasserman 1997. Separate lightcurves of Pluto and Charon. Icurus, 125(2), 233-244.

Cruikshank, D. P. and P.M. Silvaggio 1980. The Surface and Aumosphere of Pluto. Icarus, 41, 96-102.

Cruikshanl, D. P., T. L. Roush, J. Moore, M. Syles, T. C. Owen, R.H. Brown, and K.A. Tryka 1997. The surfaces of Pluto and Charon. In Pluto and Churon (S.A. Stern, and D.J. Tholen, Eds.), pp. 221-267, Univ. of arizona Press, Tucson.

Douté S., 1998. Modélisation numérique de la réflectance spectrale des surfaces glacées du Système Solaire. Application à l'analyse de spectres de Triton et Plutori et au traitement d'images hyperspectrales NDMS de lo. Thesis, LGGE - Université Paris-7, France.

Douté, S. and B. Schmitt, 1998. A multi-layer bidirectional reflectance model for the analysis of planetary surface hyperspectral images at visible and near infrared wavelengths. J. Geophys. Res., in press.

Eluszkiewicz, J., Leliwa-Kopystynski, J., Kossacki, K. J., and N. Maeno 1998. Metamorphism of solar system ices. In Solar System lces (B. Schmit, C. De Bergh, and M. Festou eds.), pp. 119-138. Astrophys. Spuce Sci. Lib., Vol. 227, Kluwer Academic Publ., Dordrecht.

Fink, U. and M.A DiSanti 1988. The separate spectra of Pluto and its satellite Charon. Astron J., 95(1), 229-236.

Gougeon, S. 1998. Contributions expérimentales à l'astrophysique en rayons X. Thesis, Université Paris-7, France.

Grundy, W. M., B. Schmit, and E. Quirico 1993. The temperature dependent spectra of alpha and beta nitrogen ice with application to Triton. Icurrs, 105, 254-258.

Grundy, W. M., and U. Fink 1996. Synoptic CCD Spectropholometry of Pluto over the past 15 years. Icarus, 124, 329-343.

Grundy, W. M., and B. Schmit 1998. The temperature-dependent near-infrared absorption spectrum of hexagonal $\mathrm{H}_{2} \mathrm{O}$ ice, J. Geophys. Res $E .103,25803-25822$.

Hapke, B. 1993. Summary of the irregular-paricle model. In Theory of reflectance und emitunce spectroscopy, 144-146, Cambridge University Press, Cambridge.

Khare, B.X., Sagan, C., Aralawa, E.T., Suits, F., Calicot, T.A. and M.W. Williams 1984. Optical constants of organic tholins produced in a simulated Titanian Atmosphere: from sofi X-ray to microwave frequencies. Icarus, 60(1), 127-137.

Lellouch, E. 1994. The thermal structure of Pluto's atmosphere: clear vs. hazy models. Icurus, 108(2), 255-264.

Lunine, J.L and D.J. Stevenson 1985. Physical state of volatiles on the surface of Triton. Nature, 317(6034), 238-240.

Owen, T.C., Roush, T.L. Cruilshank, D.P., Elliol, J.L., Young, L.A., de Bergh, C., Schmils, B., Geballe, T.R., Brown, R.H. and M.J. Bartholomew 1993. Surface ices and the atmospheric composition of Pluto. Science, 261 (5122), 745-748.

Pollack, J.B., Schwanz, J.M. and K. Rages 1990. Scalterers in Triton's atmosphere: implications for the seasonal volatile cycle. Science. $250(4979), 440-443$. 
Prokhvasilor, A. L, and L.D. Yantsevich 1983. X-ray investigation of the equilibrium phase diagram of $\mathrm{CH}_{1}-\mathrm{N}_{2}$ solid mixtures. Sov. J. Low Temp. Phys., 9, 94-98.

Quirico, E. 1995. Etudes spectroscopiques proche infrarouges de solides moléculaires. Application a l'étude des surfaces glacées de Triton et Pluton. Ph. D. dissertation, Universite Joseph Fourier, France.

Quirico, E., Schmin, B., Bini, R., and P.R. Salvi 1996. Spectroscopy of some ices of astrophysical interest: $\mathrm{SO}_{3}, \mathrm{~N}_{2}$ and $\mathrm{N}_{2}: \mathrm{CH}_{4}$ mixtures. Planet. Spuce Sci., 44(9), 973-986.

Quirico, E., and B. Schmia 1997a. Near infrared spectroscopy of simple hydrocarbons and carbon oxides diluted in solid $\mathrm{N}_{2}$ and as pure ices: implication for Triton and Pluto. Icurus, 127, 354-378.

Quirico, E, and B. Schmit 1997b. A spectroscopic study of CO diluted in $\mathrm{N}_{2}$ Ice :applications for Triton and Pluto. Icurus. 128, $181-188$.

Quirico, E., Douté, S., Schmith, B., De Bergh, C., Cruikshank, D. P., Roush, T. L. Owen, T. C., and T. R., Geballe 1998. Composition, physical state and distribution of ices at the surface of Triton. Icarus, submitued.

Roush, T.L 1994. Charon : More than water ice ?.Icarus, 108, 243-254.

Roush, T., Cruilshank, D. P., Pollack, J. B., Young, E. F. and M. J. Bartholomew 1996. Near-infrared spectral geometric albedos of Charon and Pluto: constraints on Charon's surface composition. Icurus, 119, 214-218.

Schmith, B., Quirico, E. and E. Lellouch 1992. Near infrared spectra of potential solids at the surface of Titan. Proceedings Symposium on Titan, ESA Spec. Publ., SP-338, 383-388.

Schmitu, B., S. Douté, E. Quirico, A. Benchkoura, C. de Bergh, T.C. Owen, and D.P. Cruikshank 1994. The state and composition of the surface of Pluto : Laboratory experiments and numerical modeling, Bull. Amer. Astron. Soc, 26, 1170.

Schmith, B., Quirico, E., Trotta, F. and W. M. Grundy 1998. Optical properties of ices from UV to infrared. In Solar System Ices (B. Schmilt, C. De Bergh, and M. Festou ods.), pp. 199-240. Astrophys. Space Sci. Lib., Vol. 227, Kluwer Academic Publ., Dordrecht.

Smith, E. V. P., and D. M. Gottlieb 1974. Space Sci. Rev., 16, 771-802.

Stansberry, J. A., Spencer, J. R., Schmitt, B., Benchkoura, A., Roger, V. Y. and J. I Lunine 1996. A model for the overabundance of methane in the almospheres of Pluto and Triton. Planel. Space Sci., 44 (9), $1051-1063$.

Stern, S. A., Buie, M. W. and L M. Trafton 1997. HST high-resolution images and maps of Pluto. Astron. J., 113 (2), $827-843$.

Stern, S. A. 1998. Pluto and the kuiper disk. In Solur System lees (B. Schmitt, C. De Bagh, and M. Festou eds.), pp. 685-695. Astrophys. Spuce Sci. Lib., Vol. 227, Kluwer Academic Publ., Dordrecht.

Strobel, D.F., Xun Zhu, Summers, M.E. and M.H. Stevens 1996. On the vertical thermal structure of Pluto's atmosphere. Icurus, 120(2), 266289.

Trafion, L. and S.A. Stem 1983. On the global distribution of Pluto's atmosphere. Astrophys. J., 267(2), 872-881.

Trafion, L. A. 1990. Two-component volatile aumosphere for Pluto. I. The bulk hydrodynamic escape regime. Astrophys. J., 359 (2), $512-523$.

Tryka, K. A., Brown, R. H., Cruikshank, D. P., Owen, T. C., Geballe T. R., and C. DeBergh 1994. Temperature of nitrogen ice on Pluto and its implications for flux measurements. Icurus, 112, 513.527.

Yelle, R.V. and J.I. Lunine 1989. Evidence for a molecule heavier than methane in the aumosphere of Pluto. Nuture, 339(6222), 288-290. Young, L. A. 1994. Bull: Properies and Atmospheric Structure of Pluto and Charon, Ph. D. dissertution, Massachusselts Inst. of Technology. 


\section{TABLES}

TABLE I

The different parameters of the basic surface representations $R_{\mathbf{q}}, \mathbf{R}_{\mathbf{q}}$ and $\mathbf{R}_{\boldsymbol{q}}$

\begin{tabular}{|c|c|c|}
\hline $\begin{array}{c}\text { First basic representation } \mathbf{R}_{\mathbf{i}} \\
\text { (granular mixture) }\end{array}$ & $\begin{array}{c}\text { Second basic representation } \mathbf{R}_{\mathbf{s}} \\
\text { (stratification) }\end{array}$ & $\begin{array}{c}\text { Third basic representation } \mathbf{R}_{\mathbf{g}} \\
\text { (geographical mosaic) }\end{array}$ \\
\hline $\begin{array}{l}\mathrm{G}_{\mathrm{c}}\left[\mathrm{CH}_{4}\right] \text { : } \\
\text { - number proportion } \wp_{c} \text { of } \\
\text { pure } \mathrm{CH}_{4} \text { grains } \\
\text { - mean grain size } \mathrm{r}_{\mathrm{c}} \\
\text { - scattering parameter } \mathrm{g}_{\mathrm{c}}\end{array}$ & $\begin{array}{l}\mathrm{L}_{c}\left[\mathrm{CH}_{4}\right]: \\
\text { - optical depth } \tau_{c} \\
\text { - mean grain size } \mathrm{r}_{\mathrm{c}} \\
\text { - scattering parameter } \mathrm{g}_{\boldsymbol{c}}\end{array}$ & $\begin{array}{l}\mathrm{U}_{\mathrm{c}}\left[\mathrm{CH}_{4}\right] \text { : } \\
\text { - surface proportion } \Phi_{\mathrm{c}}^{\text {surf }} \\
\text { - mean grain size } \mathrm{r}_{\mathrm{c}} \text { of pure } \\
\mathrm{CH}_{4} \\
\text { - scattering parameter } \mathrm{g}_{\mathrm{c}}\end{array}$ \\
\hline $\begin{array}{l}\mathrm{G}_{\mathrm{m}}\left[\mathrm{N}_{2}-\mathrm{CH}_{4}-\mathrm{CO}\right] \text { : } \\
\text { - number proportion } \wp_{\mathrm{m}} \text { of the } \\
\text { molecular mixture grains } \\
\text { - concentrations } \mathrm{CCH}_{4} \text { and } \mathrm{CCO} \\
\text { of } \mathrm{CH}_{4} \text { and } \mathrm{CO} \text { in the } \mathrm{N}_{2} \\
\text { matrix } \\
\text { - mean grain size } \mathrm{r}_{\mathrm{m}} \\
\text { - scattering parameter } \mathrm{g}_{\mathrm{m}}\end{array}$ & $\begin{array}{l}\mathrm{L}_{\mathrm{m}}\left[\mathrm{N}_{2}-\mathrm{CH}_{4}-\mathrm{CO}\right]: \\
\text { - concentrations } \mathrm{C}_{\mathrm{CH}} \text { and } \mathrm{CCO} \\
\text { of } \mathrm{CH}_{4} \text { and } \mathrm{CO} \text { in the } \mathrm{N}_{2} \\
\text { matrix } \\
\text { - mean grain size } \mathrm{r}_{\mathrm{m}} \\
\text { - scattering parameter } \mathrm{g}_{\mathrm{m}}\end{array}$ & $\begin{array}{l}\mathrm{U}_{\mathrm{m}}\left[\mathrm{N}_{2}-\mathrm{CH}_{4}-\mathrm{CO}\right] \text { : } \\
\text { - surface proportion }\left(1-\Phi^{\mathrm{surf}}{ }_{\mathrm{m}}\right) \\
\text { - concentrations } \mathrm{C}_{\mathrm{CH} 4} \text { and } \mathrm{COO} \\
\text { of } \mathrm{CH}_{4} \text { and } \mathrm{CO} \text { in the } \mathrm{N}_{2} \\
\text { matrix } \\
\text { - mean grain size } \mathrm{r}_{\mathrm{m}} \\
\text { - scattering parameter } \mathrm{g}_{\mathrm{m}}\end{array}$ \\
\hline
\end{tabular}


TABLE II

Three unit surface representation $R_{s c n}$ and its best fit parameter values
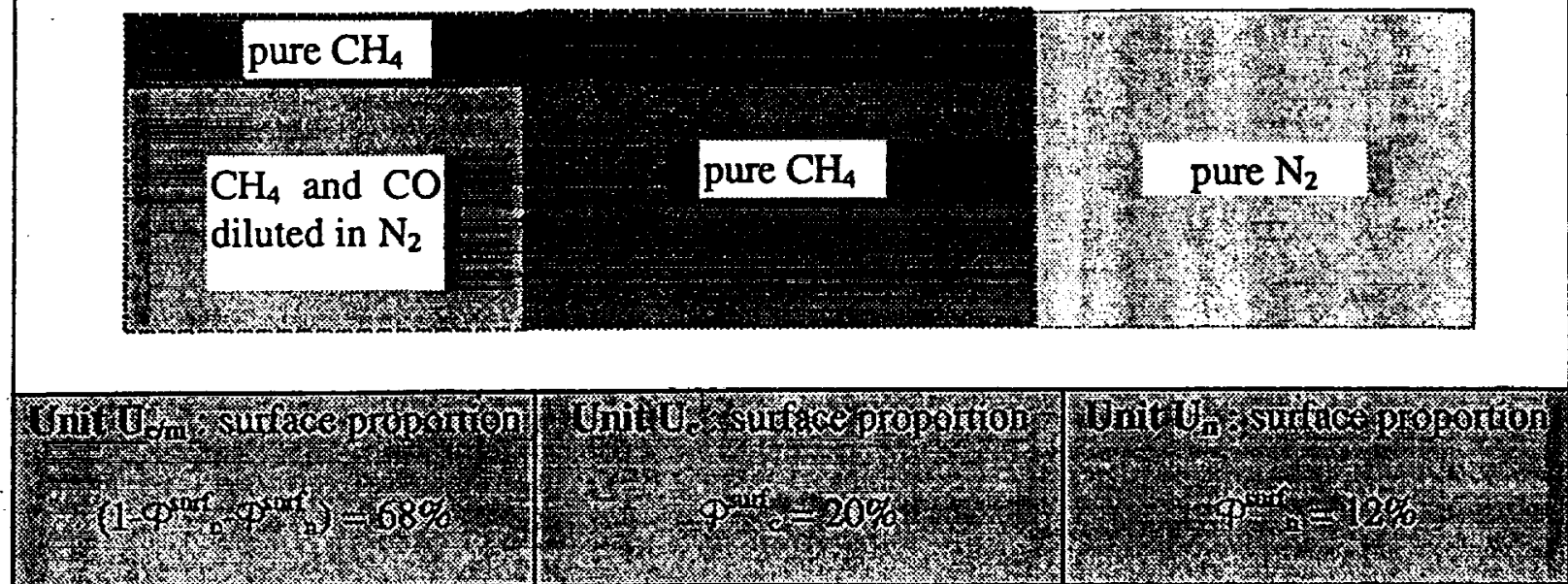

\section{$\mathrm{L}_{c}\left[\mathrm{CH}_{4}\right]:$}

$\left[\mathrm{CH}_{4}\right]$ :

$\left[\mathrm{N}_{2}\right]$ :

- optical depth $\tau_{c}=0.3$

- mean grain size $r_{c}=500 \mu m$

- mean grain size $r_{n}=10 \mu m$

- mean grain size $r_{c s}=100 \mu \mathrm{m}$

- scattering parameter $\mathrm{g}_{c}=0$

- scattering parameter $g_{n}=0$

- scattering parameter $\mathrm{g}_{c s}=0$

$\mathrm{L}_{m}\left[\mathrm{~N}_{2}-\mathrm{CH}_{4}-\mathrm{CO}\right]:$

- concentrations

$\mathrm{C}_{\mathrm{CH} 4}=0.45 \% ; \mathrm{C}_{\mathrm{CO}}=$ $0.08 \%$

- mean grain size

$$
\mathrm{d}_{\mathrm{m}}=5.510^{4} \mu \mathrm{m}
$$

- scattering parameter $g_{m}=0$ 
TABLE III

Three unit surface representation $R_{-\infty}-$ and its best fit parameter values

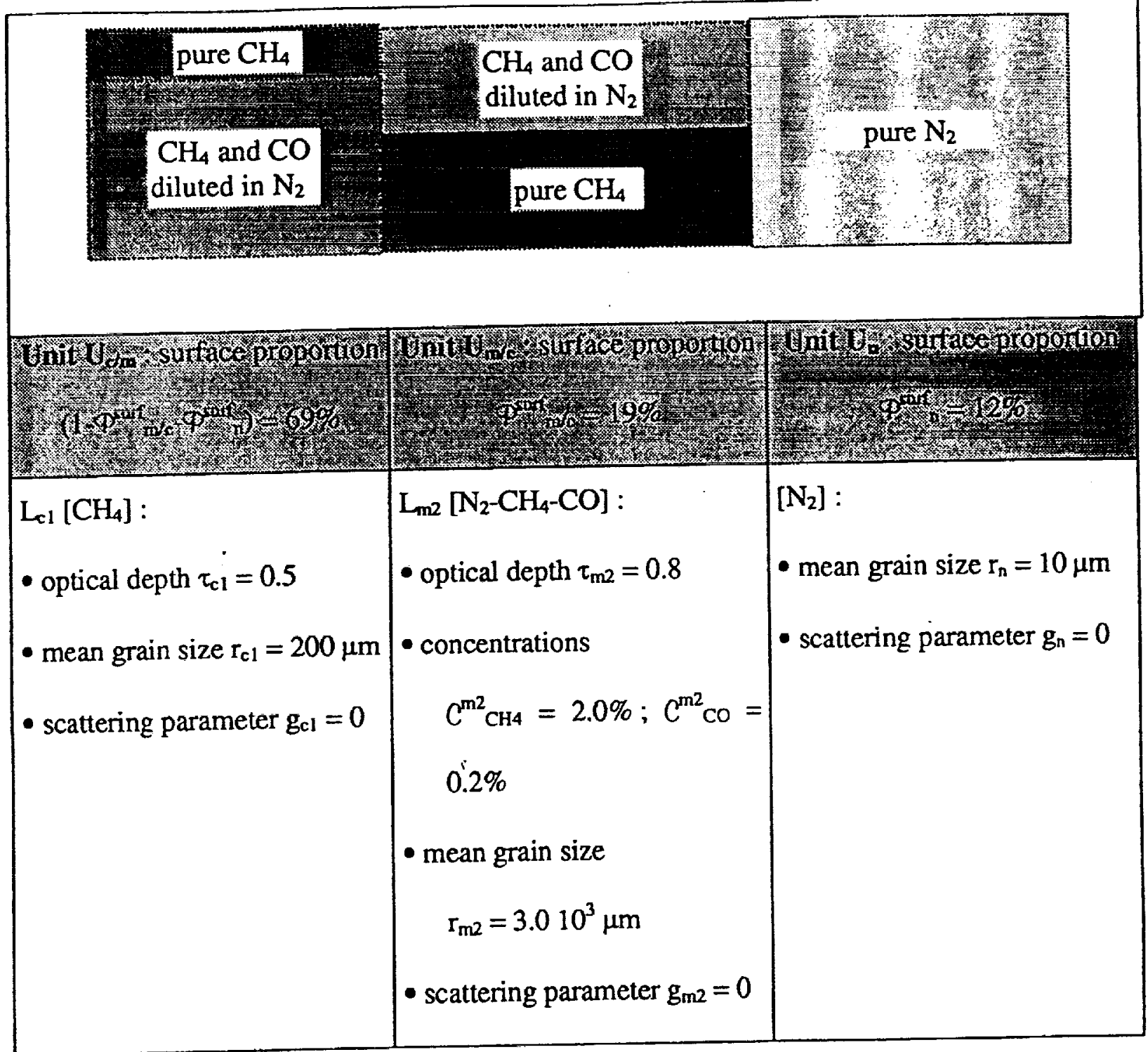




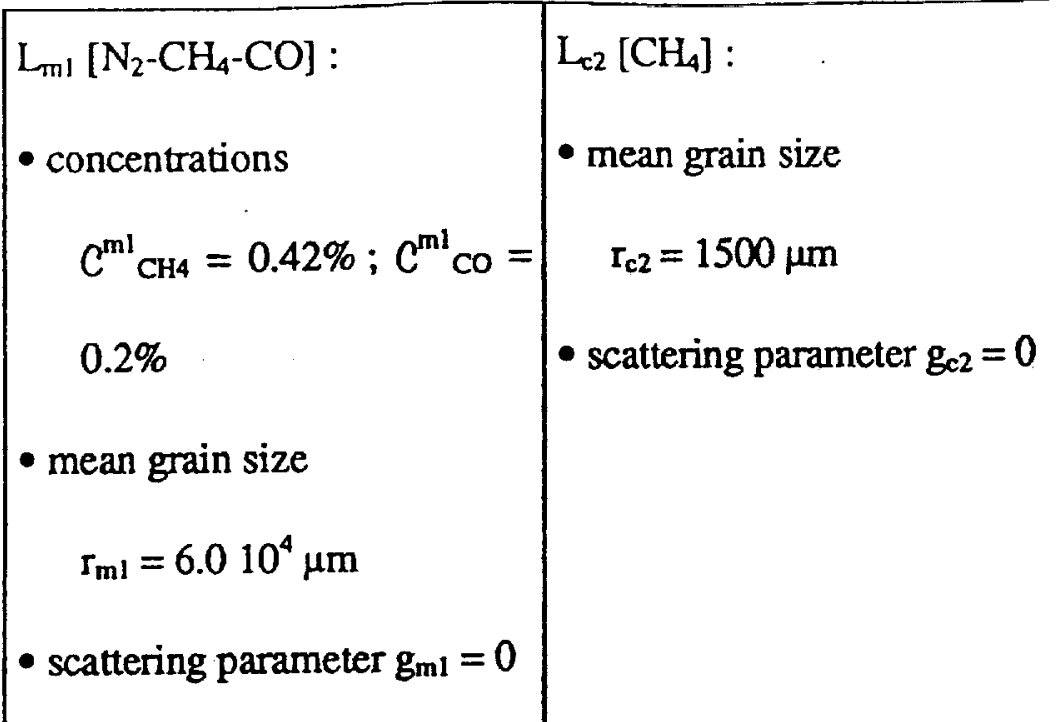


TABLE IV

Three unit surface representation $R_{\mathrm{mcn}}$ and its best fit parameter values

\begin{tabular}{|c|c|c|}
\hline 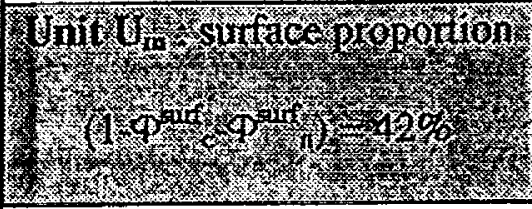 & 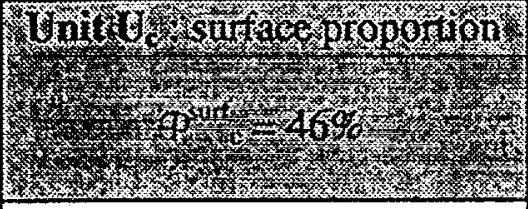 & 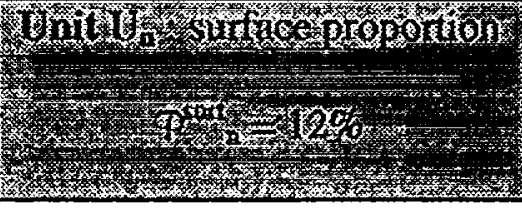 \\
\hline$\left[\mathrm{N}_{2}-\mathrm{CH}_{4}-\mathrm{CO}\right]$ : & $\mathrm{rCH}^{-}$ & $N_{n}$ \\
\hline concentrations & - mean orain cize $r=200 u m$ & - mean orain size $r=10 u m$ \\
\hline Coirceriu audins & 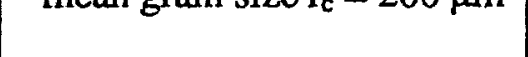 & 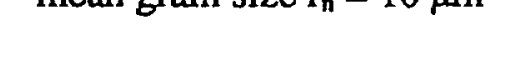 \\
\hline$C_{\mathrm{CH} 4}=0.3 \% ; C_{\mathrm{CO}}=0.1 \%$ & - scattering parameter $g_{c}=0$ & - scattering parameter $\mathrm{g}_{\mathrm{n}}=0$ \\
\hline - mean grain size & & \\
\hline$r_{m}=9.510^{4} \mu \mathrm{m}$ & & \\
\hline - scattering parameter $g_{m}=0$ & & \\
\hline & & \\
\hline & & \\
\hline
\end{tabular}




\section{FigURE CAPTIONS}

FIG. 1.: Visible geometric albedo light curve of the Pluto-Charon as calculated by Buie et al . (1997). The location of Pluto (sub-Earth longitude) during the three 1995 UKIRT/CGS4 spectrophotometric observations, the 1992 and 1994 observations used to check our calibration operation, as well as at the time of the eclipse observation of Charon in 1987, are indicated.

FIG. 2. : Calibration operation applied to the $05 / 11 / 95$ observation of Pluto. The $H$ region (dotdash line) is adjusted to the $\mathrm{K}$ region by a multiplication factor to form a new coherent spectrum (solid line). This spectrum is compared to better calibrated spectra from 05/27/92 and 04/16/94 at similar rotational phases (dotted and dashed lines).

FIG. 3. : Low resolution modeling (solid line) of the spectral geometric albedo of Charon calculated by Roush et al. (1996) (dashed line), from the 04/23/87 mutual event observations (Buie et al. 1987). In this model $60 \%$ of the surface is covered by $\mathrm{H}_{2} \mathrm{O}$ ice (grain size $50 \mu \mathrm{m}$ ) and the remaining by a spectrally neutral component.

FIG. 4. : Geometric albedo of the Pluto-Charon system from the 15 May observation (dotted line), of Charon (dashed line) at high resolution calculated by fitting the low resolution observations (see FIG. 3), and of Pluto alone (solid line) after extraction of the Charon contribution to the total flux.

FIG. 5. : Identification of the absorption bands and illustration of the choice of the significant characteristic spectral features on the spectrum of Pluto that are good criteria for the estimation of the modeling quality. a) $\mathrm{H}$ region, b) $\mathrm{K}$ region. 
FIG. 6. : Comparison of the 1995 albedo spectra (a: H region; b: K region) of Pluto with laboratory transmission spectra of pure $\mathrm{CH}_{4}$ ice at $40 \mathrm{~K}$ (band positions indicated by vertical dotted lines) and of $\mathrm{CH}_{4}$ diluted in $\beta-\mathrm{N}_{2}$ ice at $40 \mathrm{~K}$ (positions : vertical dot-dash lines). The spectrum of Triton (09/07/95) is also shown for comparison (from Quirico et al. 1998). All spectra are shifted vertically for clarity.

FIG. 7. : Two 'best' simulations for a simple geographical mosaic of a neutral unit $U_{n}$ (fine grained $\mathrm{N}_{2}$ ) and a molecular $\mathrm{N}_{2}-\mathrm{CH}_{4}-\mathrm{CO}$ mixture $\left(\mathrm{R}_{\mathrm{mn}}\right)$ or pure $\mathrm{CH}_{4}\left(\mathrm{R}_{\mathrm{cn}}\right)$.

FIG. 8. : The 'best' simulation for a simple geographical mosaic $\left(R_{i n}\right)$ of a neutral unit $U_{n}$ (fine grained $\mathrm{N}_{2}$ ) and an intimate (granular) mixture of pure $\mathrm{CH}_{4}$ grains and $\mathrm{N}_{2}-\mathrm{CH}_{4}-\mathrm{CO}$ molecular mixture grains $\left(\mathrm{U}_{\mathrm{c}+\mathrm{m}}\right)$.

FIG. 9. : Surface representation $R_{\text {scn }}$ : the best fit of this representation to the spectrum of Pluto. The best fit parameter values are reported in TABLE II.

FIG. 10. : Surface representation $R_{s s^{-}}$: the best fit of this representation to the spectrum of Pluto. The best fit parameter values are reported in TABLE III.

FIG. 11. : Surface representation $R_{m e n}$ : the best fit of this representation to the spectrum of Pluto. The best fit parameter values are reported in TABLE IV.

FIG. 12. : Comparison between the simulations corresponding to the representations $R_{s c n}$ and $R_{s s}{ }^{-} n$ (see text). a) $\mathrm{H}$ region, b) $\mathrm{K}$ region.

FIG. 13. : Comparison between the simulations corresponding to the representations $R_{s s}-2$ and $R_{m e n}$ (see text). a) $\mathrm{H}$ region, b) $\mathrm{K}$ region. 
FIG. 14. : A possible 'scenario' of the sublimation and re-condensation processes which affect the ices and the surface state of Pluto, as suggested by our modeling. 


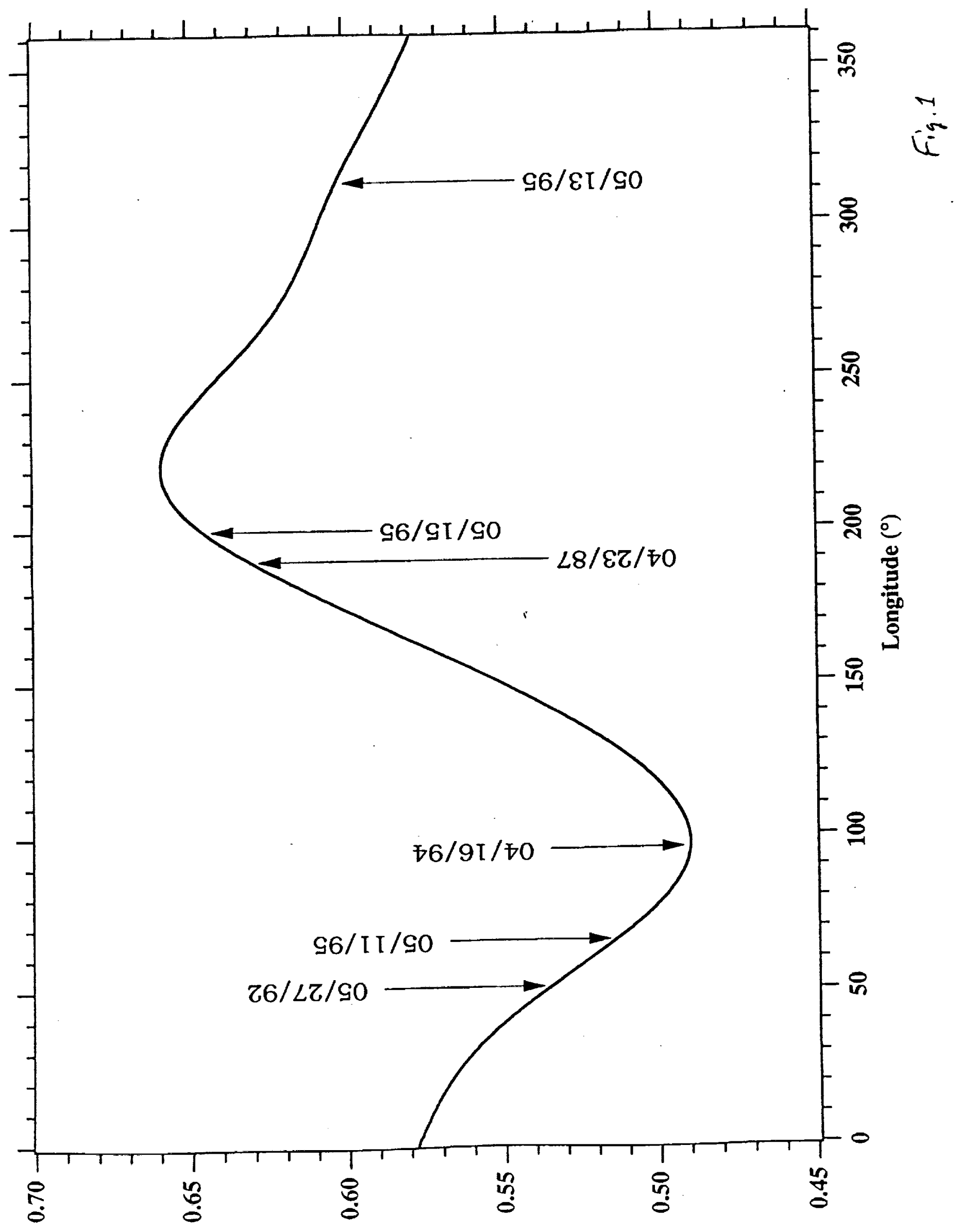




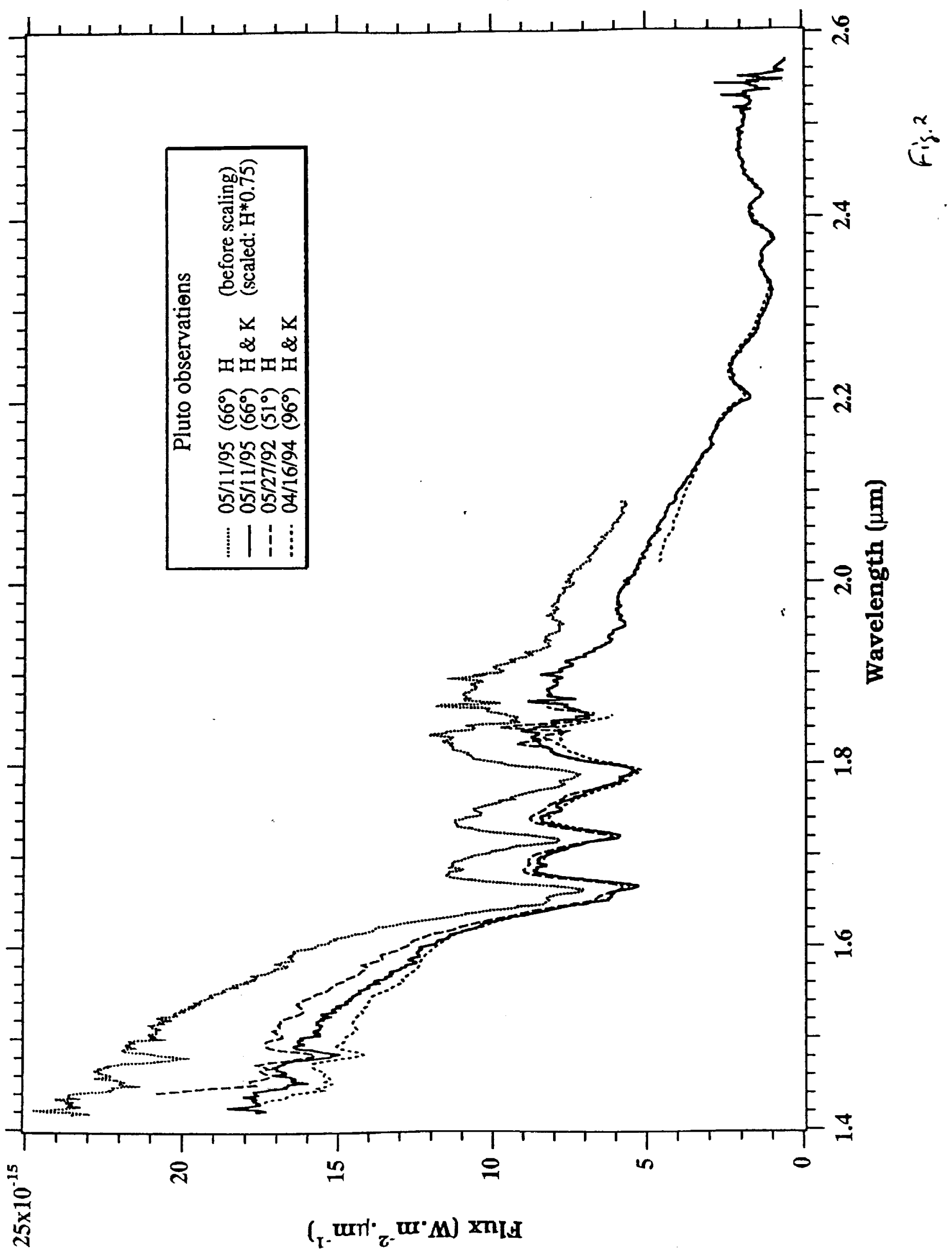




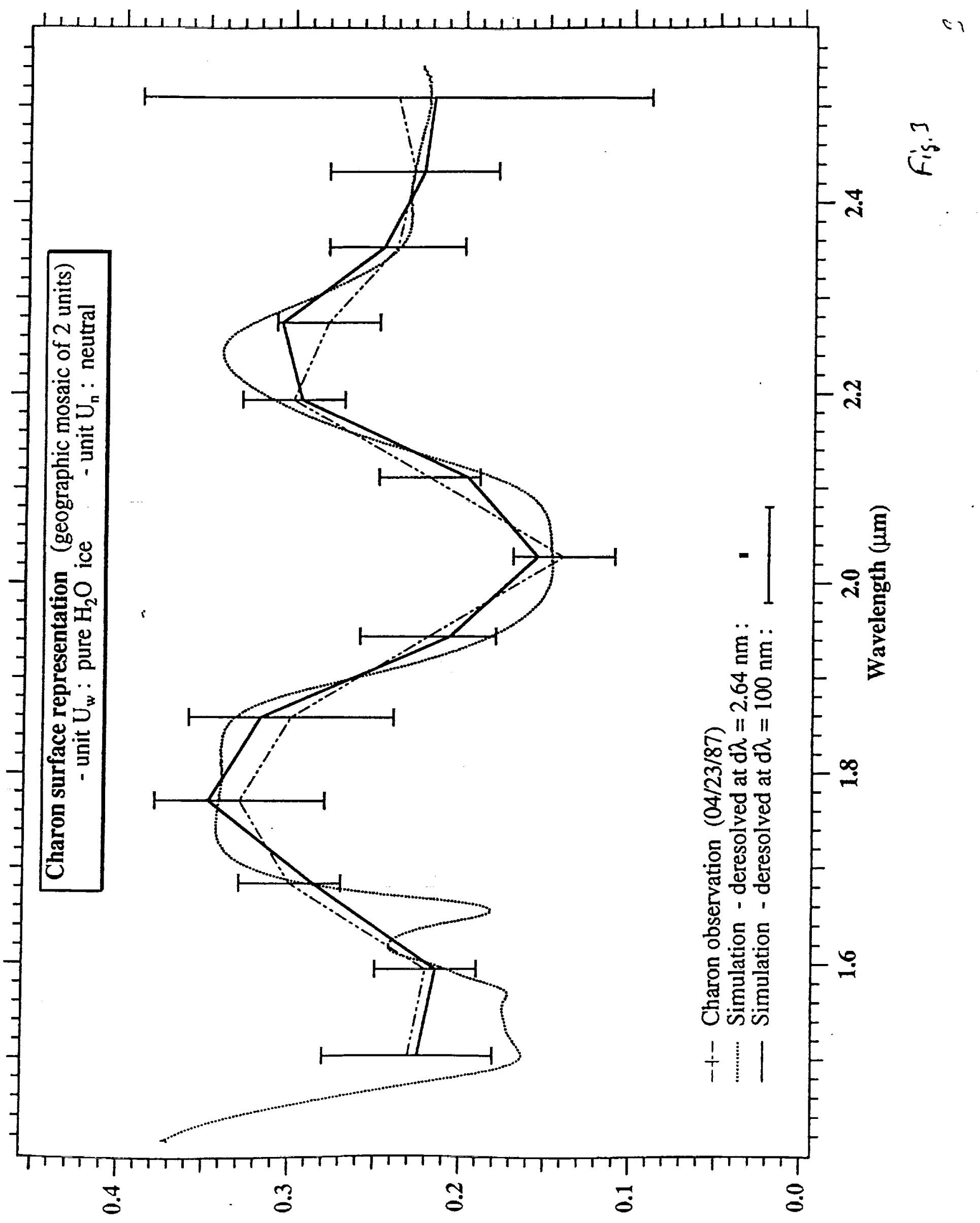




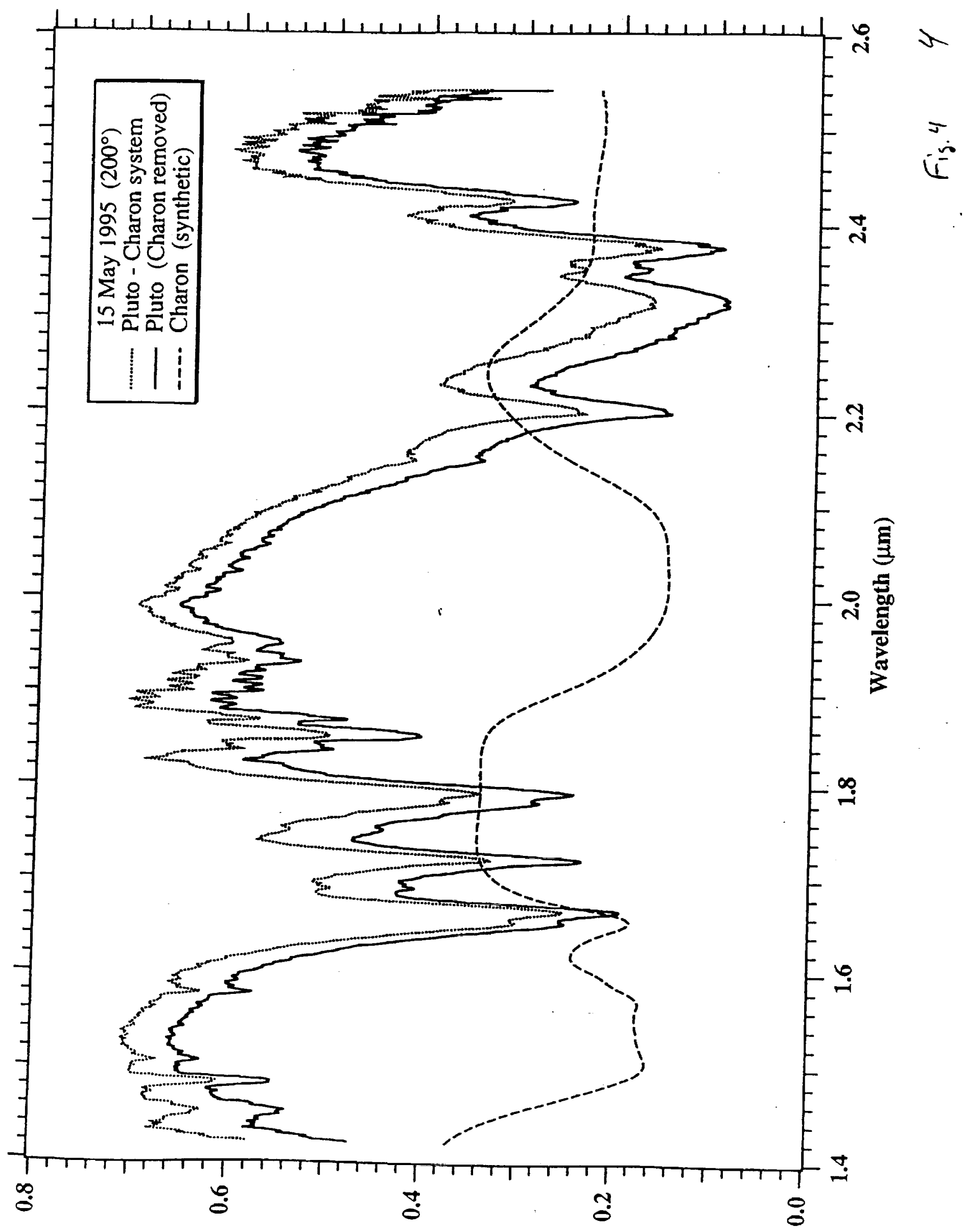




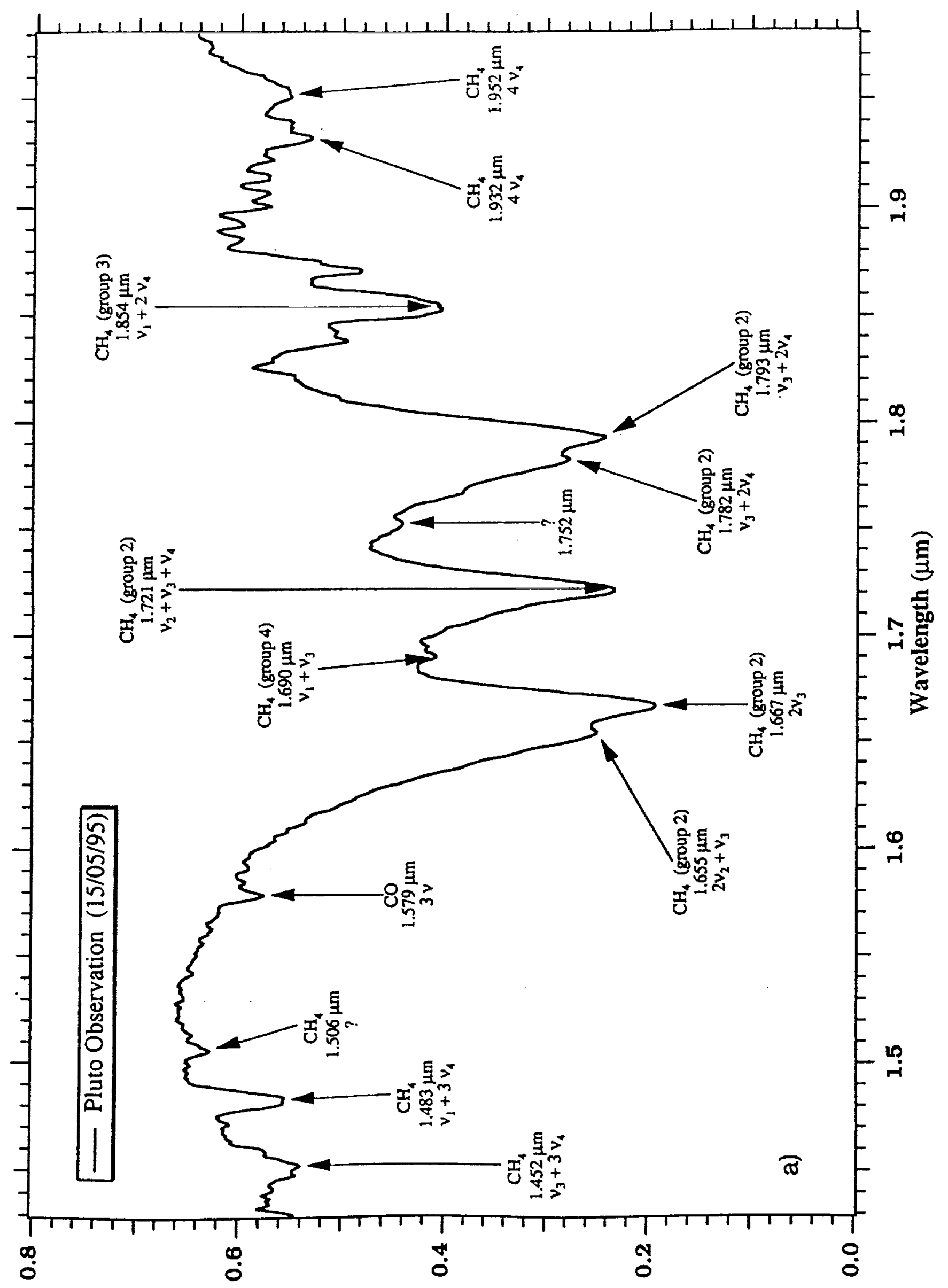

onadry axramnan 


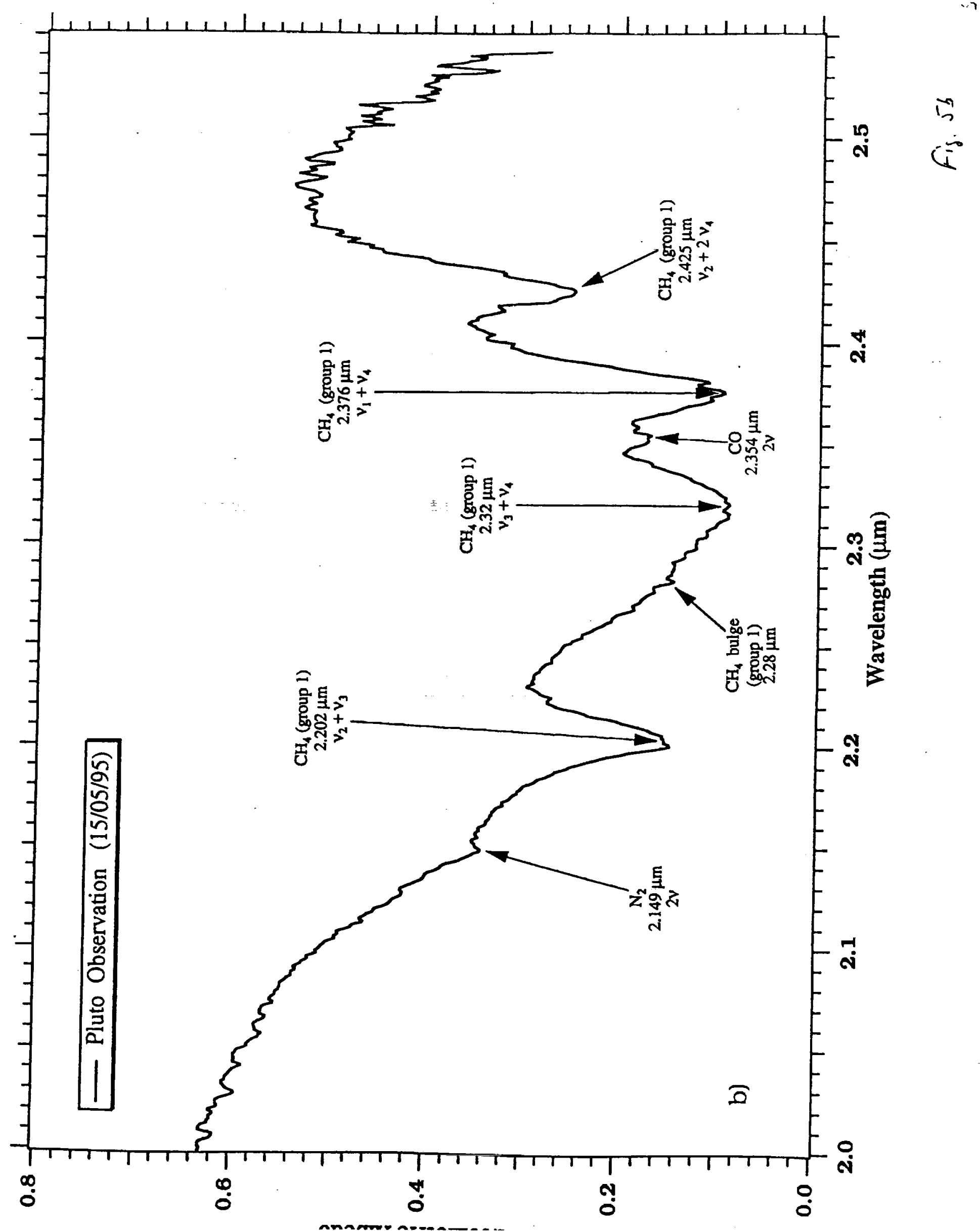




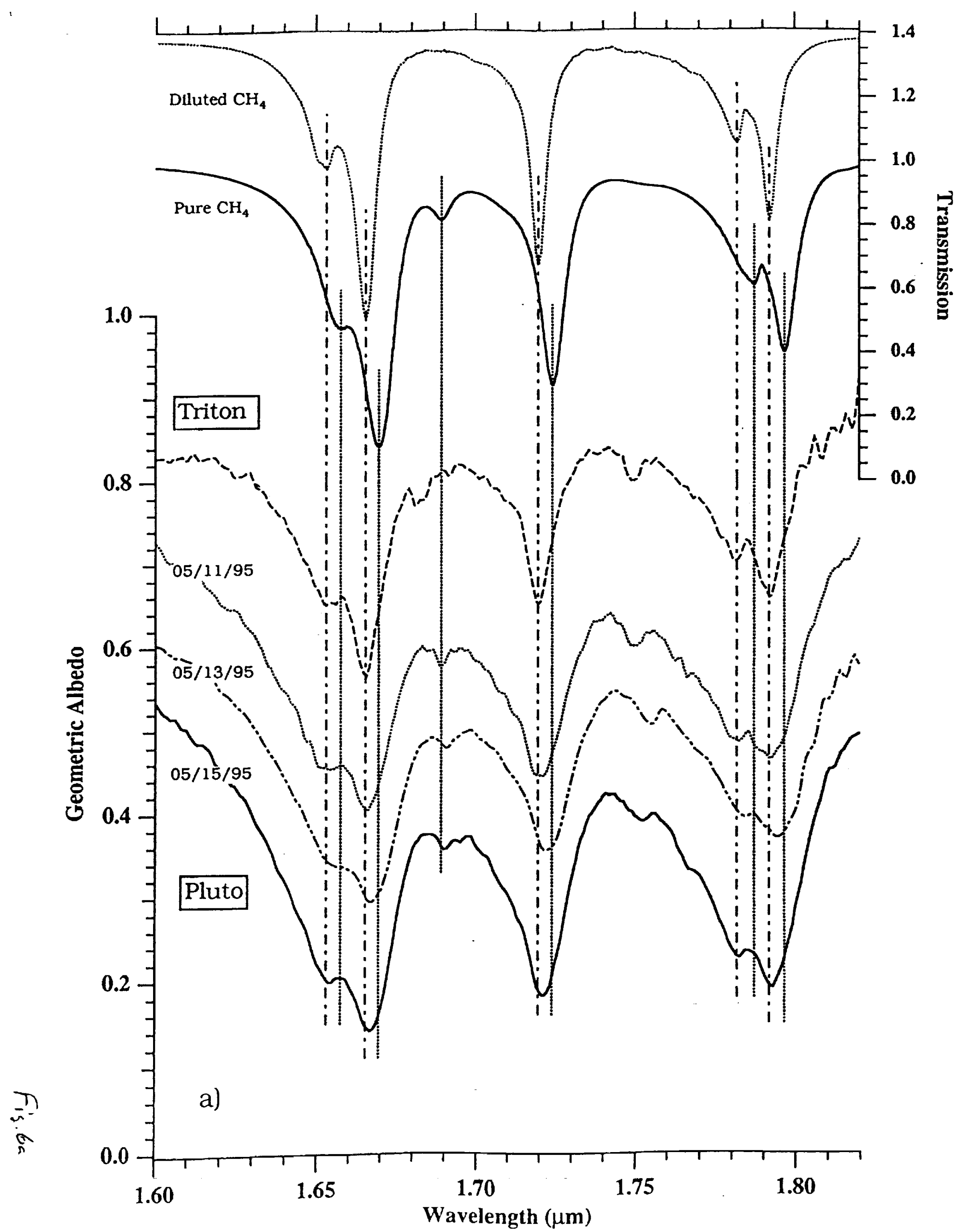




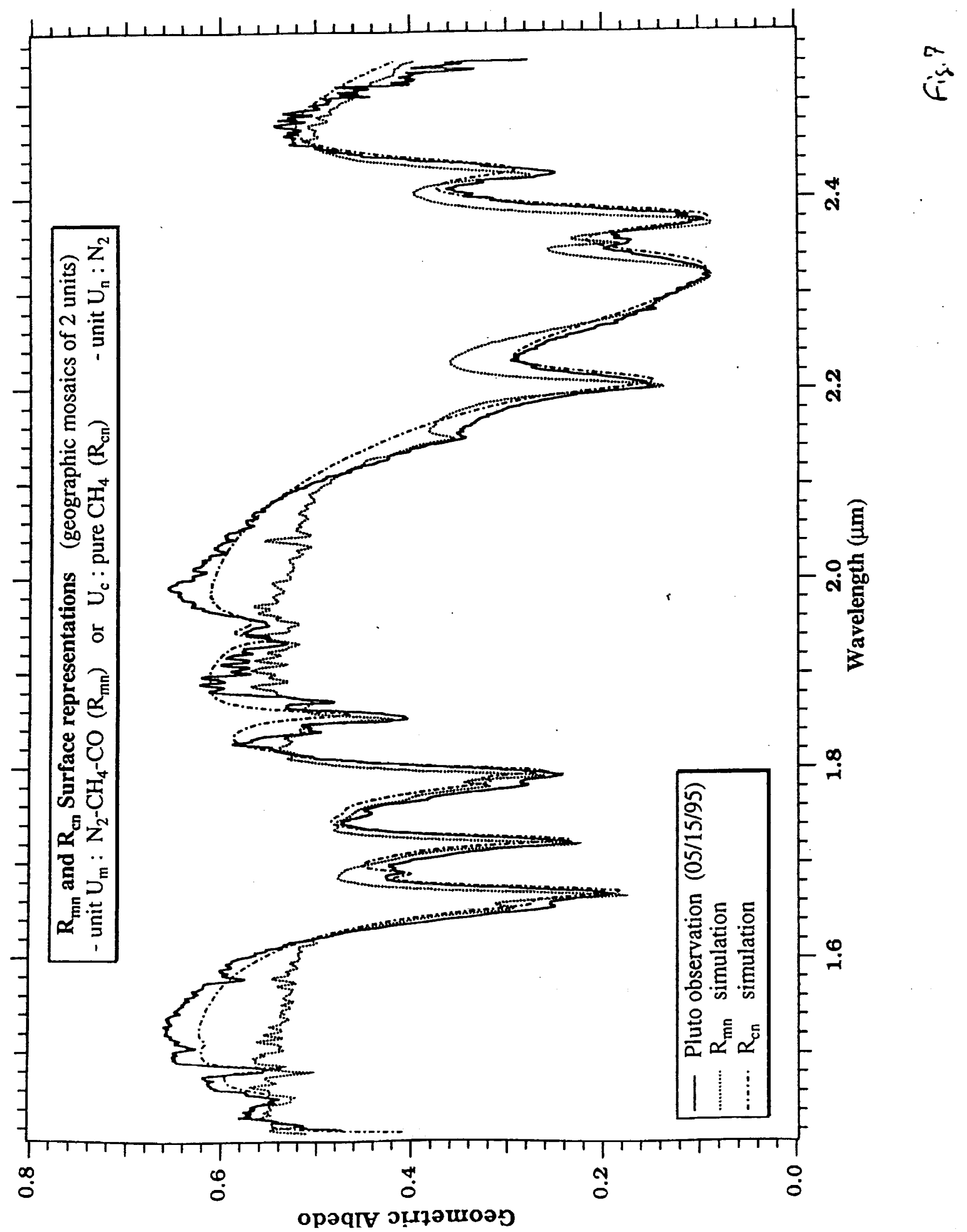




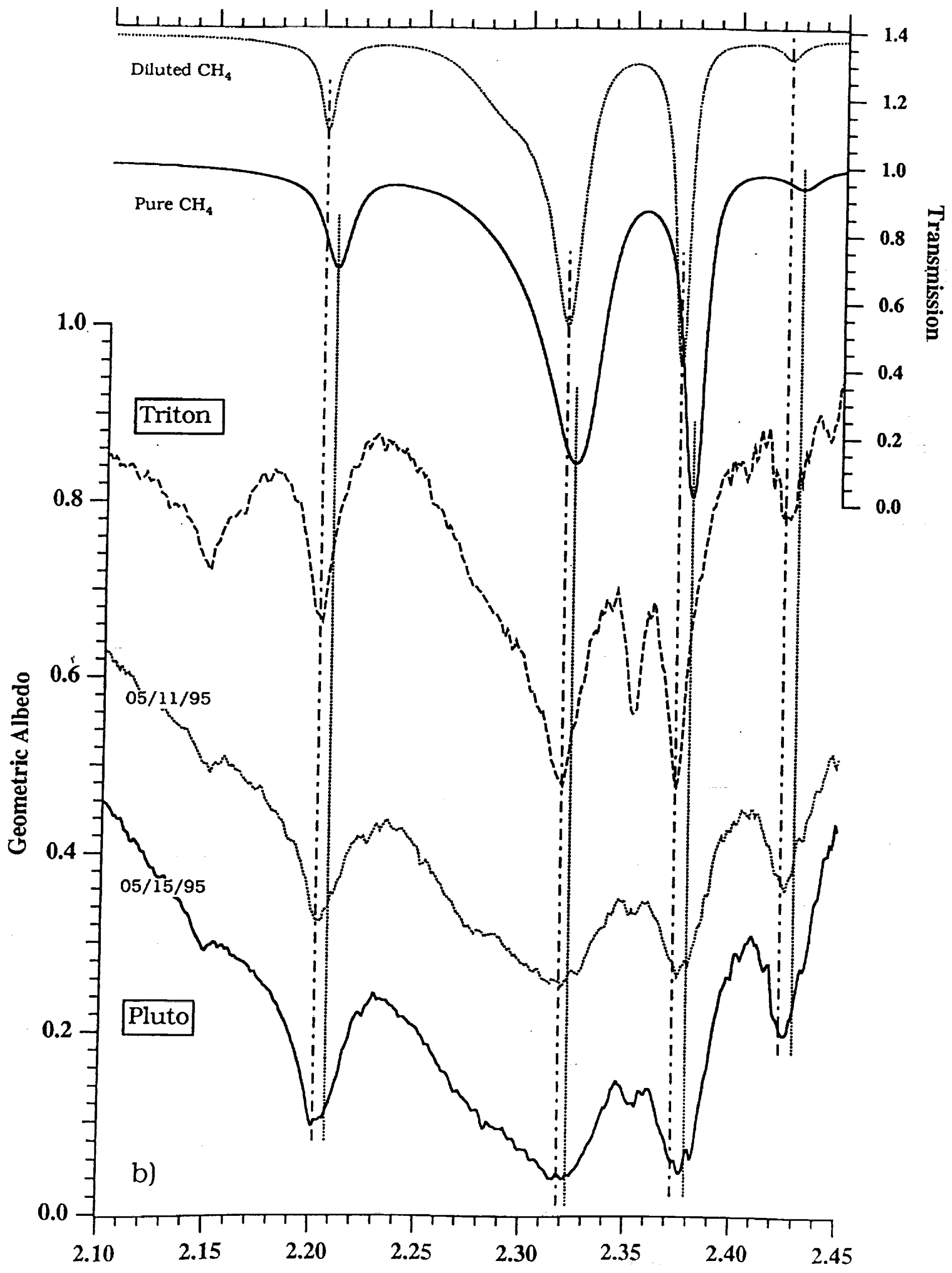




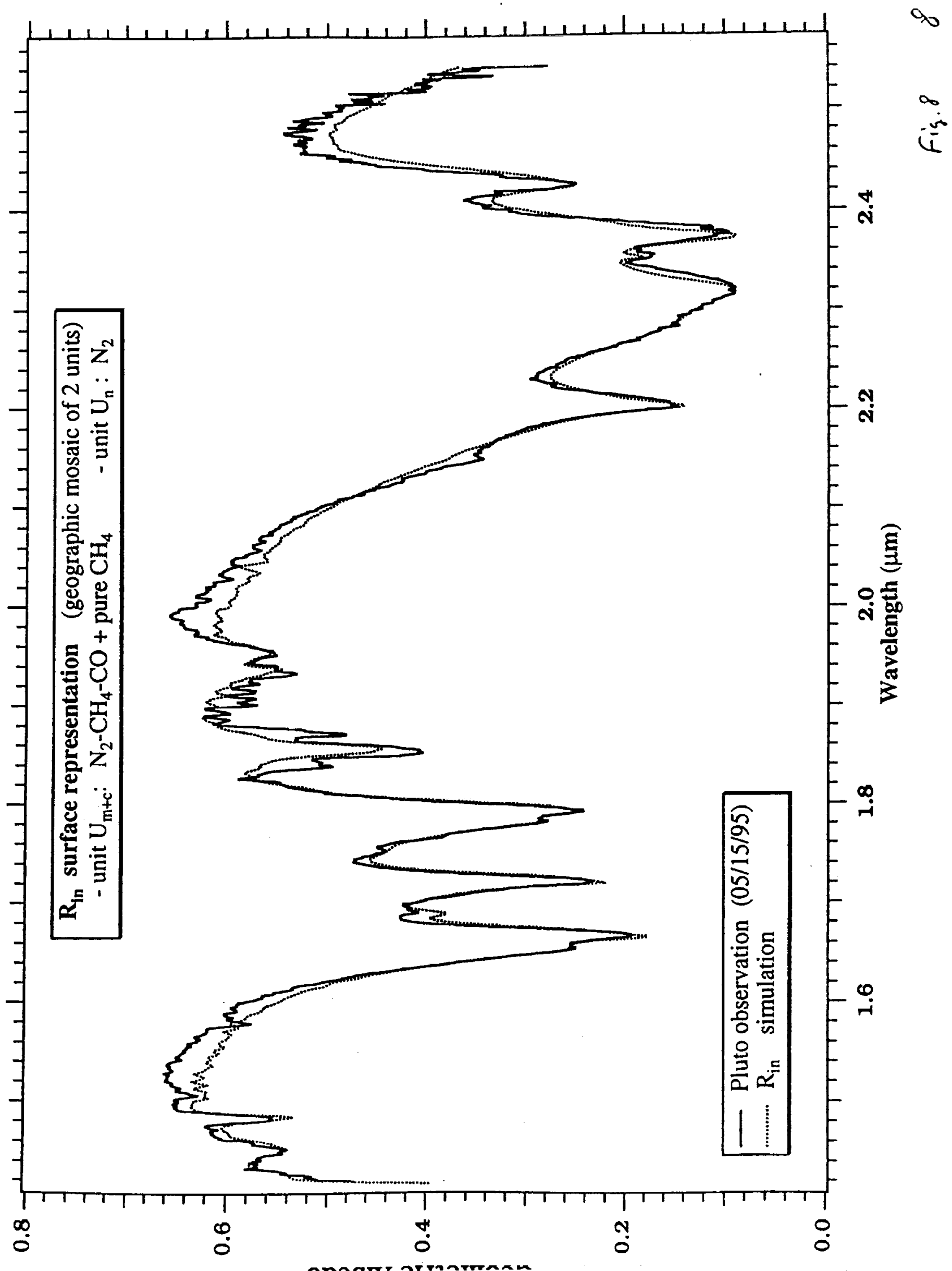




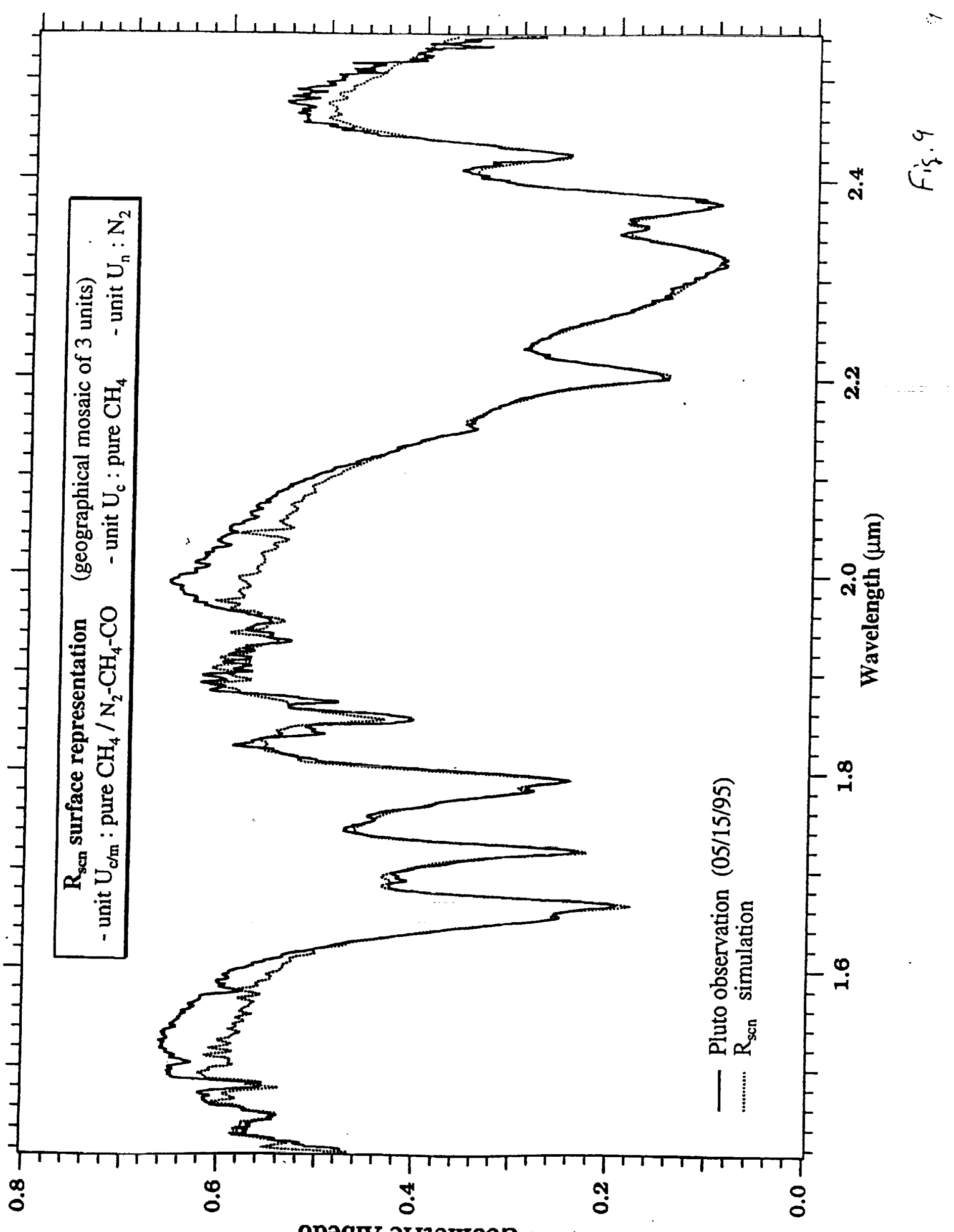




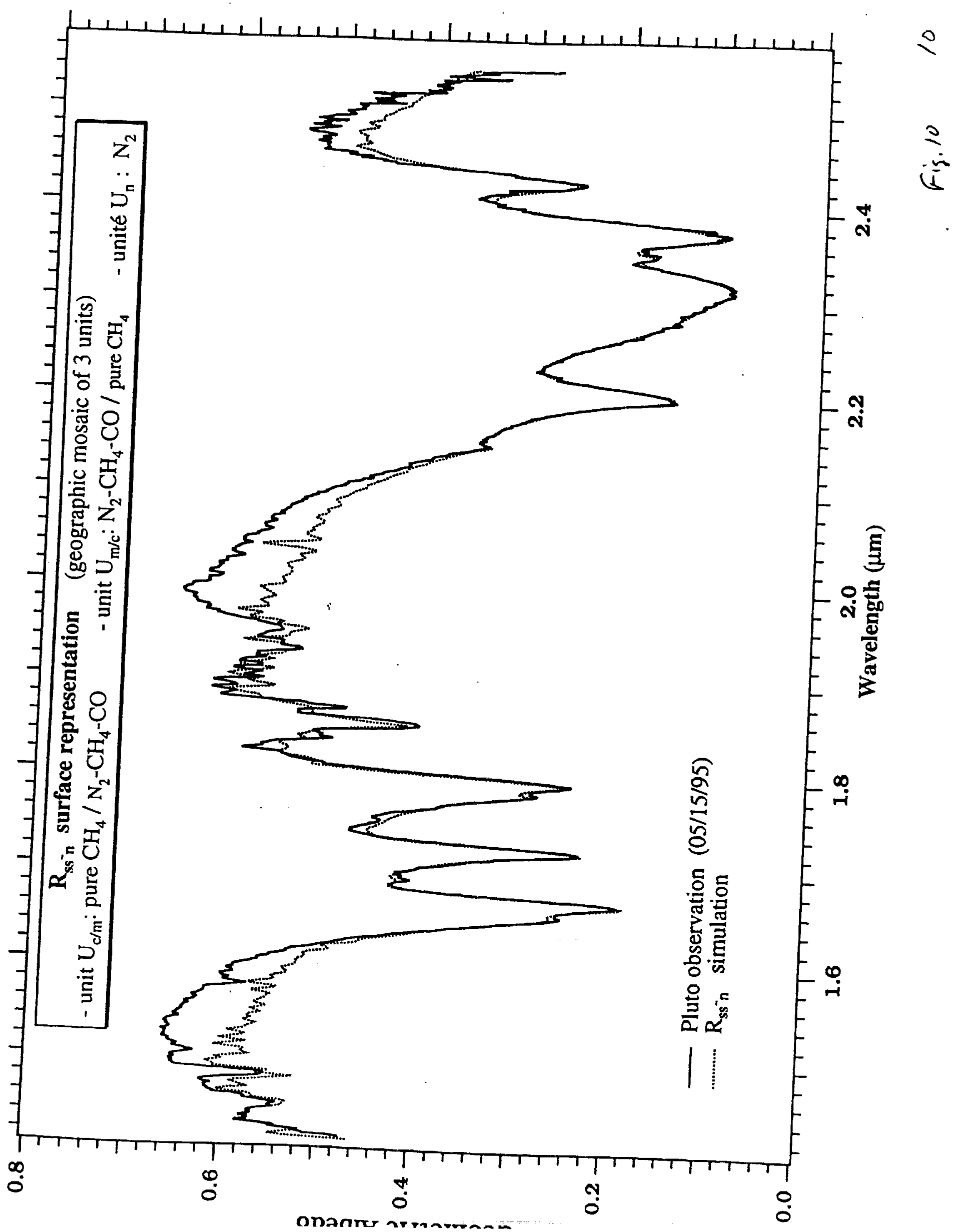




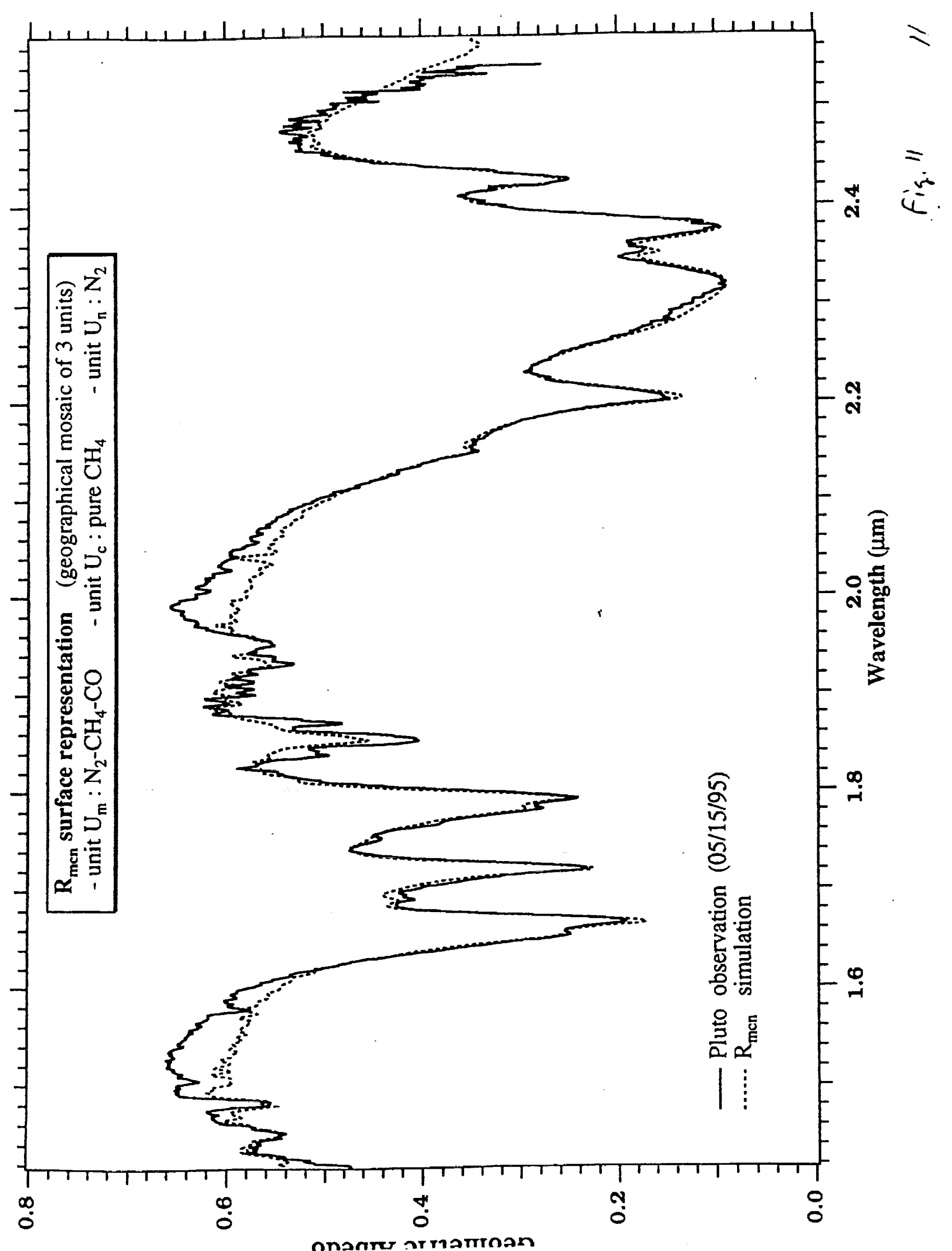




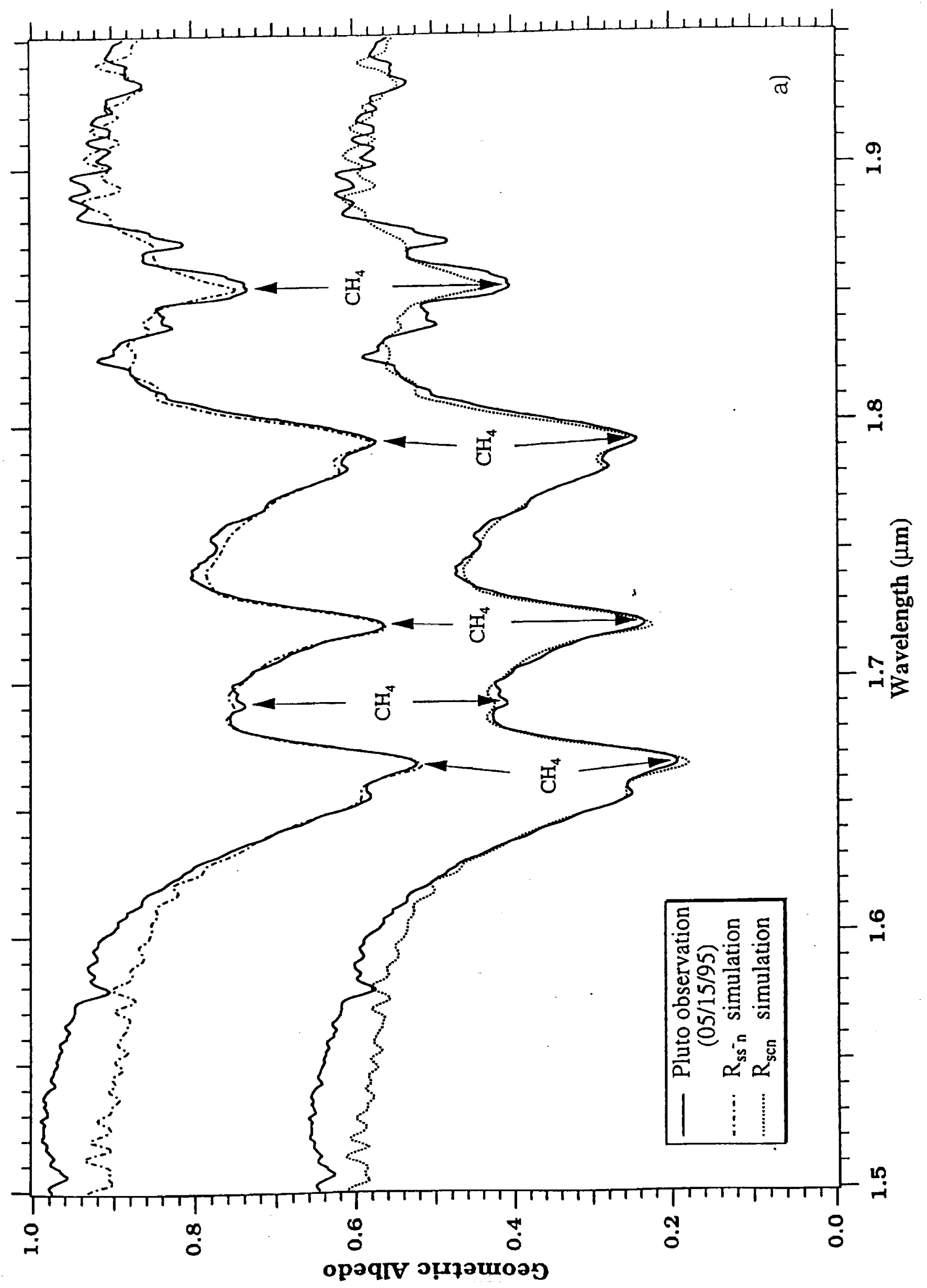




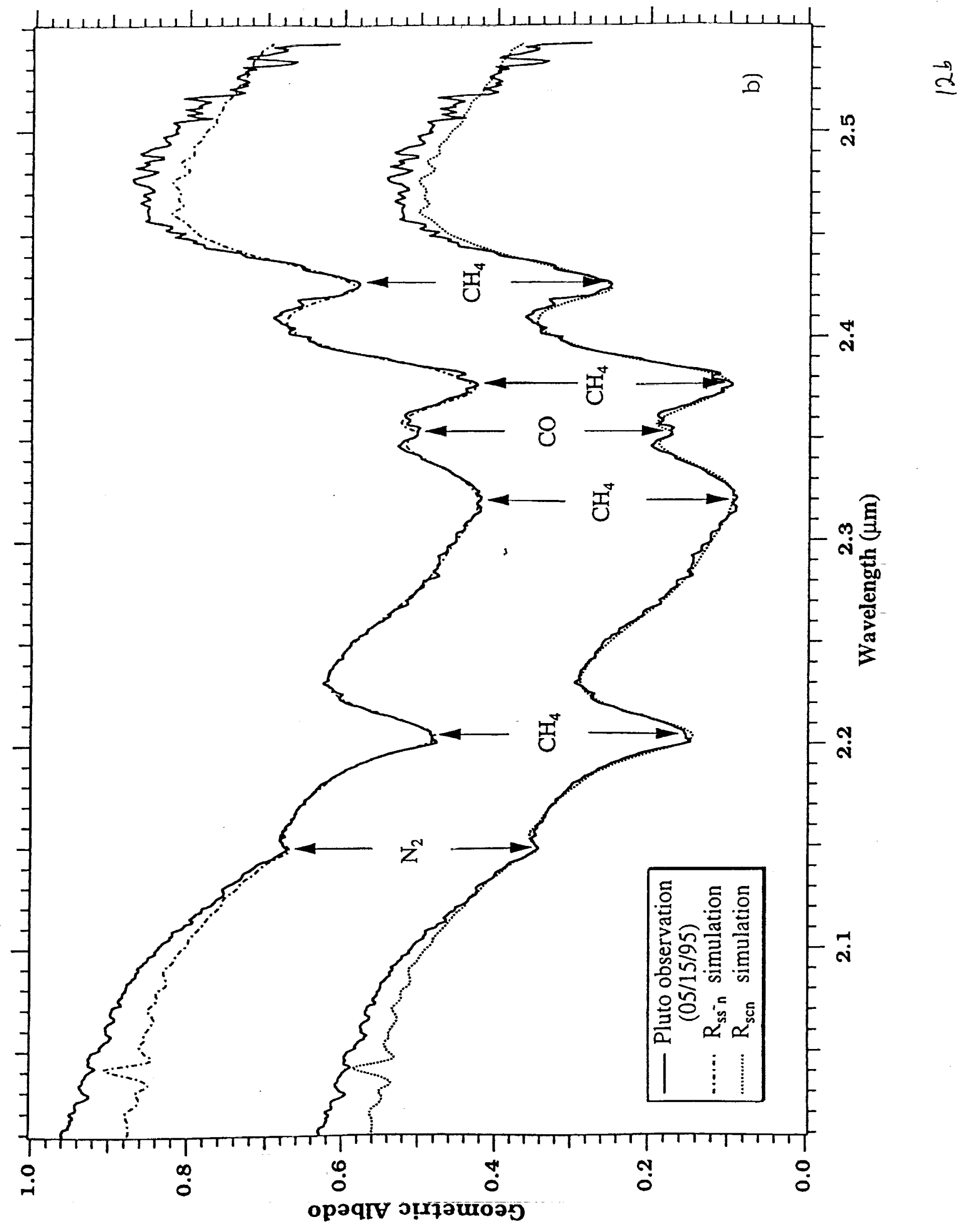




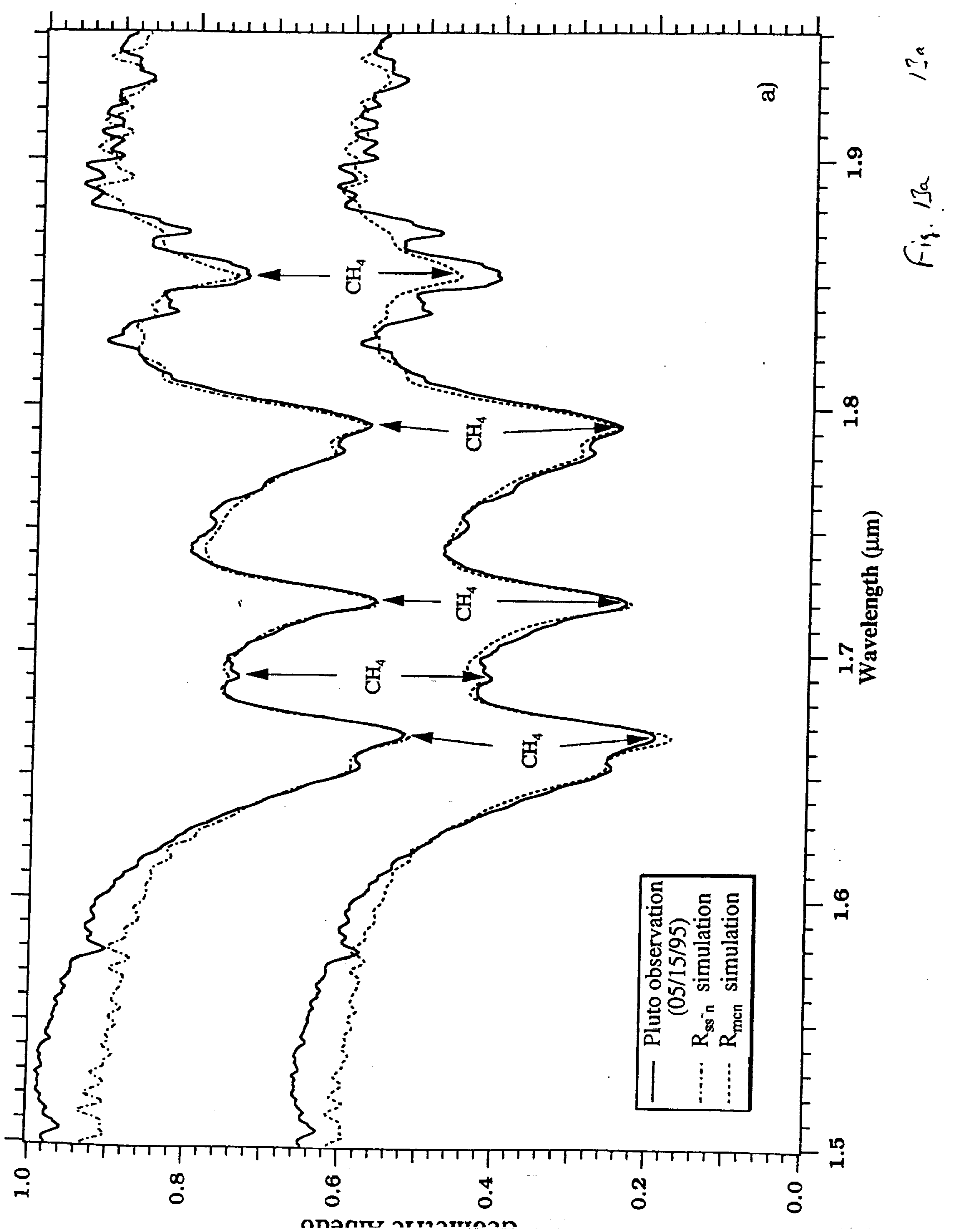




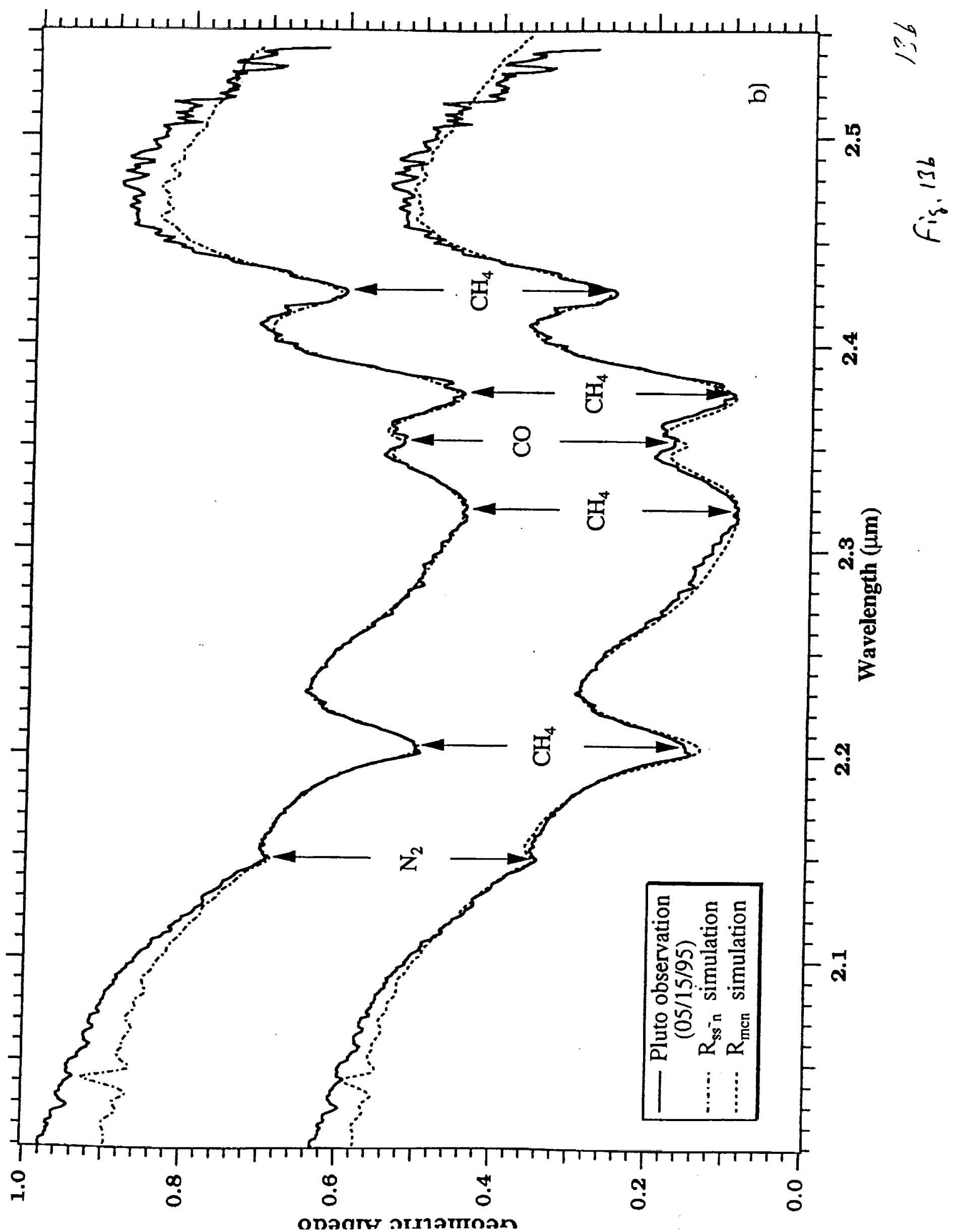




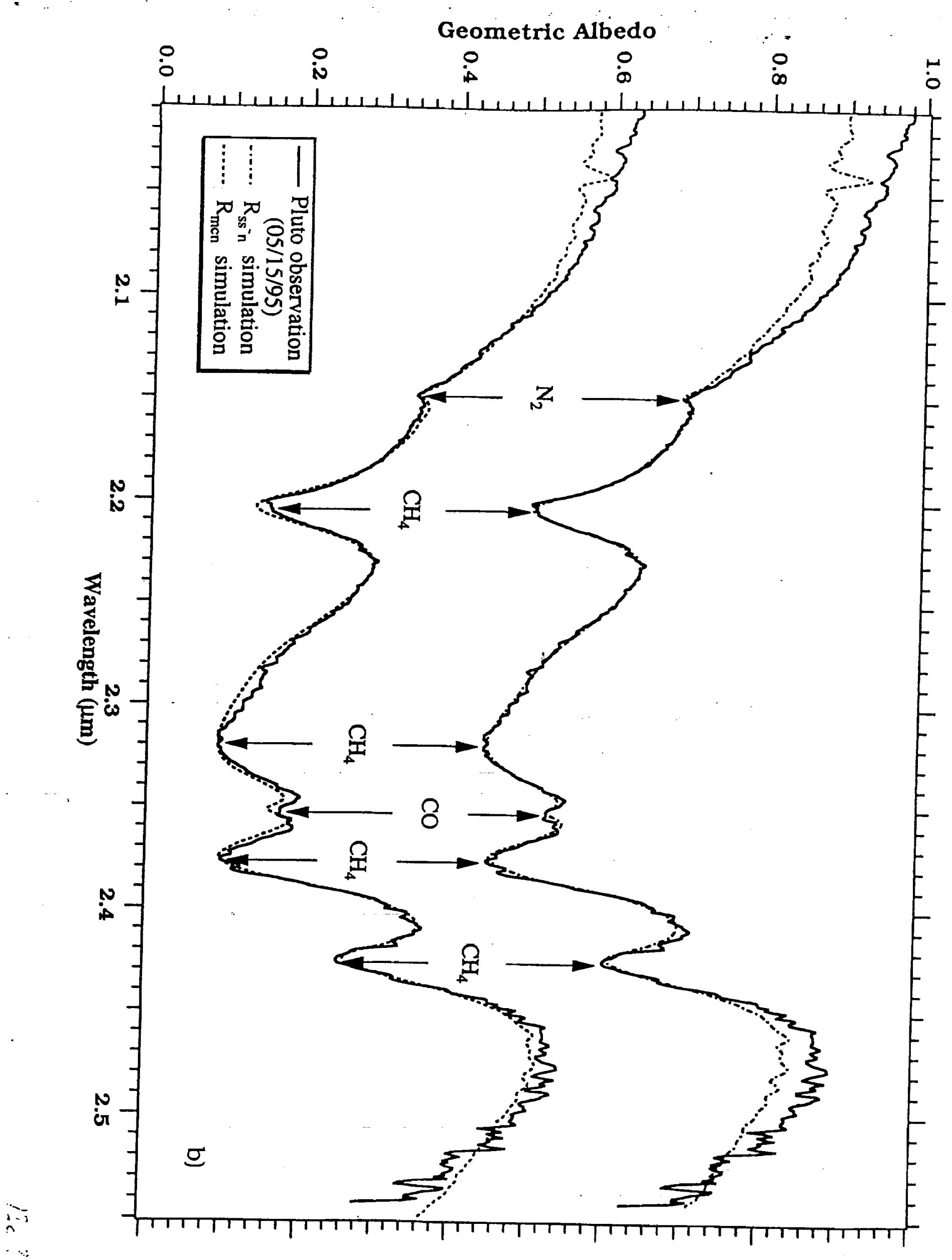




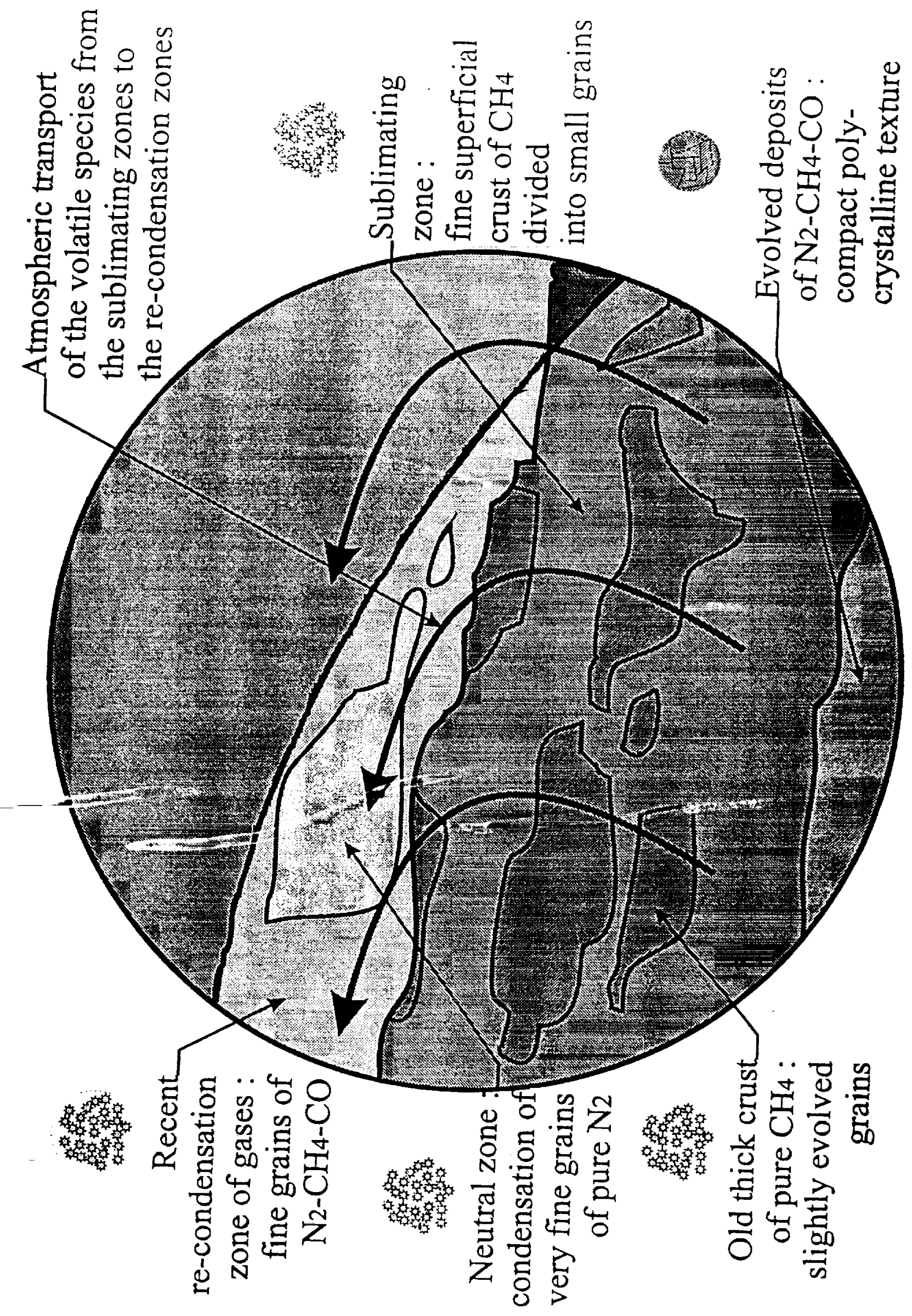

